\title{
Cardiovascular imaging 2019 in the International Journal of Cardiovascular Imaging
}

\author{
Johan H. C. Reiber ${ }^{1} \cdot$ Gabriel T. R. Pereira $^{2} \cdot$ Luis A. P. Dallan $^{2} \cdot$ Hiram G. Bezerra ${ }^{2}$. Johan De Sutter ${ }^{3}$. \\ Arthur E. Stillman ${ }^{4}$ Nico R. L. Van de Veire ${ }^{5}$ Joachim Lotz ${ }^{6}$
}

Published online: 13 April 2020

(c) Springer Nature B.V. 2020

Please, find below an overview of the most relevant papers in the International Journal of Cardiovascular Imaging over the year 2019 for the different modalities. Overall, a great number of very interesting papers have been published.

\section{X-ray imaging}

In the year 2019 many great manuscripts were published in the field of X-ray angiography, which are highlighted in the following paragraphs:

Karabag and his team studied the possible relationship between serum uric acid (SUA) and the presence of coronary artery disease as described by the SYNTAX score II (SSII) and subsequent cardiovascular events in a population of 290 patients with multi-vessel disease and/or unprotected left main disease who were treated by PCI [1]. They concluded that the SUA level was associated with high SSII and longterm mortality in this patient population.

Rigatelli and colleagues assessed the performance and 3-years outcome od unprotected left main (LM) stenting using either the Culotte or a novel nano-crush technique in

Johan H. C. Reiber

J.H.C.Reiber@lumc.nl

1 Division of Image Processing, Department of Radiology, Leiden University Medical Center, Leiden, The Netherlands

2 Department of Cardiology, Case Western Hospitals, Cleveland, OH, USA

3 Department of Cardiology, AZ Maria Middelares Gent and University Gent, Gent, Belgium

4 Department of Radiology, Emory University Hospital, Atlanta, GA, USA

5 Department of Cardiology, AZ Maria Middelares Gent and Free University Brussels, Brussels, Belgium

6 Department of Radiology, University Medical Center Göttingen, Göttingen, Germany a population of 65 patients ( 32 nano-crush and 33 Culotte) in this single-center study [2]. The nano-crush patients showed lower contrast medium volume and X-ray exposure, shorter fluoroscopy and procedures time compared to the Culotte technique. Also at a mean FU-time of 27.4 months, MACE were lower for the nano-crush technique.

An in-vivo comparison between 2D QVA angiography, 3D QVA and intravascular ultrasound in peripheral angiography in 30 porcine femoral angiograms was carried out by Zasada et al. [3]. The overall conclusion was that IVUS and 3D QVA compared very well and that the luminal diameters were greater than by standard 2D QVA. They concluded from this study, that 3D QVA is a useful surrogate of IVUS for precise luminal morphology measurements of peripheral arteries.

In the paper by van Es et al., a novel stand-alone software tool was presented and validated for image guided cardiac ablation therapy in an animal study including 10 pigs [4]. The new CARTBox 2 software enables therapy target selection based on infarction transmurality and local wall thickness measurements derived from preoperative MRI scans. The workflow and results were compared with the commercially available NOGA system. The data from this study demonstrated that the procedures performed with CARTBox 2 were equally accurate and quicker than with the NOGA system. On the other hand, the CARTBox 2 requires acquisition of an LGE-MRI scan and more fluoroscopy time.

Another paper on radiofrequency catheter ablation (RFCA) was presented by Dengke et al., who investigated the Ensite NavX 3D mapping system in left accessory pathway (AP) disorders [5]. A total of 227 patients had their AP treated by RFCA and who were subdivided into a study group of 112 patients and 115 patients in the control group. The concluded that the irradiation time and exposure dose together with the total operation duration was significantly reduced with the Ensite mapping system in comparison with the traditional X-ray fluoroscopy method. 
Fan et al. investigated the feasibility of low frame rate fluoroscopy during primary PCI for patients with acute ST elevation myocardial infarction (STEMI) [6]. The total of 165 consecutive patients were divided into two groups: fluoroscopy at 7.5 frames/s and fluoroscopy at 15 frames/s. They found that the 7.5 frames/s group was associated with $48.9 \%$ reduction in X-ray dose area product, and a $61.1 \%$ reduction in the air kerma, while there were no differences in procedure time, fluoroscopy time, and contrast volume. Also, the new protocol did not increase the incidence of contrast-induced nephropathy, nor peri-PCI cumulative adverse events. As a result, they concluded that the low frame rate fluoroscopy at 7.5 frames/s is a safe and feasible strategy for reducing radiation during PCI.

The 12-month clinical results of the SeQuent ${ }^{\circledR}$ Please DCB drug-coated balloon for patients with de novo coronary lesions in vessels exceeding $3.0 \mathrm{~mm}$ was studied by Liu et al. in a total of 120 patients; this is a single center prospective observational study [7]. The 12-month incidence of target lesion failure (TLF) was $3.4 \%$, and clinically driven target vessel revascularization (TLR) was also $3.4 \%$; however, coronary dissection occurred in 42 patients (35\%), two of which underwent bail-out stent implantation. They conclude that the study shows the feasibility, initial safety and efficacy of coronary intervention with this stent; further, the coronary dissection occurs post DCB treatment occurs frequently, but is safe at follow up.

Zhang et al. validated a new approach for automatic coronary blood flow computation from coronary angiograms to further automate the Quantitative Flow Ratio (QFR) approach for the assessment of the physiologic significance of coronary vessels [8]. A total of 328 vessels with paired FFR data from the FAVOR II China study was used for the validation. The flow velocity computed by the new approach had a weak to moderate correlation with the manual frame count method; on the other hand, the QFR auto had a good correlation $(r=0.96)$ with the conventional QFR. The areaunder-the-curve in ROC analysis by both approaches did not differ significantly. They concluded that the automatic computation of patient-specific coronary flow velocity based on coronary angiography is feasible.

The relationship of pre-procedural Dmax based sizing to lesion level outcomes in Absorb BVS and Xience EES treated patients in the AIDA trial was described by the team of Tijssen [9]. Lesion-oriented outcomes (LOCE) that occurred during 2 years of $\mathrm{FU}$ were related to device non-oversized or oversized status as related to the QCA pre-procedure; the total number of treated lesions available for DMax analysis was 2152. The investigators concluded that there was no significant difference in LOCE between oversized and non-oversized treated ABSORB BVS and Xience EES treated lesions. The majority of very late scaffold thrombosis occurred in properly sized devices.
The team of Kishima et al. investigated predictors of left atrial appendage (LAA) stunning after electrical cardioversion in patients with atrial fibrillation [10]. The patient population included 134 patients who underwent catheter ablation for non-paroxysmal, non-valvular, and symptomatic atrial fibrillation (AF). They found that the rate of LAA-stunning was the highest in the group with high brain natriuretic peptide (BNP) and long-lasting AF.

Right ventricular (RV) systolic dysfunction is prognostic in various cardiovascular diseases and not commonly assessed in the catheterization laboratory. Baumann et al. developed a novel and reproducible method based on the measurement of maximum RCA movement (distance and speed) to assess RV systolic function during selective coronary angiography [11]. The validation was done in 97 consecutive patients and compared with tricuspid annular plane systolic excursion as measured by echocardiography. A cut-off value less than $22.3 \mathrm{~mm}$ systolic RCA motion had a specificity of $93.3 \%$ and a sensitivity of $75.6 \%$ for identifying an abnormal right ventricular systolic function.

$\mathrm{Wu}$ et al. studied the combination of fractional flow reserve (FFR) and superficial wall stress (SWS) in a new one-way fluid-structure interaction (FSI) [12]. They performed a total of 54 analyses in virtual coronary lesion models based on plaque compositions, arterial remodelling patterns, and stenosis morphologies under physiological conditions. Four types of lesions were identified based on the combination of cutoff values for FFR (0.80) and maximum relative SWS $(30 \mathrm{kPa})$. The level of risk was the highest for lipid-rich plaques with severe stenosis and withoutto-positive remodelling.

The team of Üveges studied the relationship between the 3D spatial morphology of stented coronary segments and restenosis [13]. Sixty-four patients, 27 of them with BM stent and 37 with DES) were investigated retrospectively $12 \pm 6$ months after the index procedure. The curvature of a segment was defined by a ratio of vessel length along the vessel and a straight line distance between the edge points of the stent (ACr ratio). They found that the pre-stent $\mathrm{ACr}$ was an independent predictor of in-stent restenosis. They believe that the $\mathrm{ACr}$ predispose to chronic shear stress in the vessel wall, which may contribute to the pathological intimal proliferation.

The topic of anatomical and physiologic differences in lesion characteristics after drug-coated balloon (DCB) angioplasty or metal stent placement for de novo lesions was discussed by Chung et al. [14]. They studied baseline and post-balloon parameters in a group of 151 patients with 167 lesions. Post-balloon FFR cut-off value of 0.75 was used to define functionally significant lesions. A weak correlation was found between residual \% diameter stenosis and postballoon FFR. There was no correlation between dissection severity and post-balloon FFR. In the high post-balloon 
FFR group, long-term clinical outcomes were not different between the DCB and stent groups with or without the adjustment of lesion characteristics. The authors conclude that post-balloon FFR measurements may be safe and effective compared to angiography-guided treatment if DCB only treatment is considered.

Rodriguez-Granillo et al. studied the relationship between the CAD-RADS scoring system based on computed tomography and the complexity of lesions by invasive coronary angiography (ICA) [15]. A total of 91 stable patients who underwent both CTA and ICA were included in this study. Based on the ICA findings, the Syntax score and CAD extension index were calculated. The authors found a significant relationship between the CAD-RADS and both the SYNTAX and CAD extension score, although the complexity of coronary anatomy differed among patients with CAD-RADS $\geq 4$ A.

In the manuscript by Watarai et al. the Quantitative Flow Ratio (QFR) was compared with the wire-based iFR approach in a prospective study enrolling 150 coronary lesions in consecutive patients with intermediate stenosis evaluated by iFR [16]. The mean QFR and iFR were $0.81 \pm 0.12$ and $0.89 \pm 0.11$, respectively. The area-underthe-curve of QFR predicting $\mathrm{iFR} \leq 0.89$ was 0.91 . The authors concluded that the QFR, including on-site analysis, demonstrated a good correlation with iFR and a diagnostic performance comparable to that of iFR in consecutive patients with intermediate coronary stenosis.

And finally, Hideo-Kajita et al. studied the impact of two formulas to calculate $\% \mathrm{D}$ stenosis of luminal obstructions in two data sets: ten phantom lesion models and $354 \mathrm{CAD}$ lesions from the FIRST trial were assessed by QCA [17]. The 2 approaches are: \%DS1 averages the proximal and distal reference vessel diameters; and \%DS2 interpolates the reference diameters. In the clinical study, two scenario's were assessed: scenario A using the worst view, which is the most common approach in the clinical setting; and scenario $\mathrm{B}$, the average of 2 complementary views which is the standard core-lab approach. The authors conclude that the overall means of the formulas provide similar results, but with significant lesion-level differences. Further, the use of the worst view versus the average of two views also provided similar results.

\section{Intravascular imaging}

The year of 2019 was very interesting for the intravascular imaging field, with several new technologies being investigated in addition of important definitions on stablished modalities. The International Journal of Cardiovascular Imaging published several of those topics involving all aspects of intravascular imaging. Of note, there was a great increase in the number of original articles published in 2019 compared to the previous years.

\section{Evaluation of drug-eluting stents (DES)}

Highlighting the importance of intravascular imaging, especially OCT, on guiding PCI, Tomaniak et al. [18] analyzed a series of 3- and 9-year optical coherence tomography (OCT) looking at vascular healing response to sirolimus- (SES) and paclitaxel (PES) -eluting stents including 22 subjects (8 SES and 14 PES). They found that at 3 and 9 years after PCI, implantation of early-generation SES and PES may be associated with similar neointimal thickness, strut coverage, malapposition and protrusion among patients with uneventful 3 years follow-up.

In an effort to understand the impact of intravascular imaging on clinical outcomes, Xiao-Fei et al. [19] evaluated intravascular ultrasound (IVUS) guidance in patients undergoing drug-eluting stent implantation in a robust metaanalysis of 9 randomized trials including 4724 patients, which is the largest sample-size data regarding this topic to date. They demonstrated that IVUS-guided DES implantation significantly reduced cardiac death, coronary revascularization and stent thrombosis, particularly for patients with IVUS-defined optimal procedures compared with angiography guidance.

In an attempt to characterize the impact of lesion tortuosity on OCT findings and clinical outcomes after DES implantation, Nakamura et al. [20] investigated vessel wall injuries and stent apposition as assessed by OCT after second- and newer-generation DES implantation. They included 95 de novo lesions treated with a single DES (62 platinumchromium everolimus-eluting stents and 33 bioresorbablepolymer sirolimus-eluting stents) in a 12-months follow-up. Strut-based analysis showed that angled lesions demonstrated a higher rate of malapposed strut in the distal and proximal sub-segments. The 12 month clinical outcomes were comparable between the groups. Compared to nonangled lesions, angled coronary lesions were associated with a higher incidence of intra-stent dissection and incomplete stent apposition on post-intervention OCT after implantation of second- and newer-generation DESs.

\section{Evaluation of ST-segment elevation myocardial infarction (STEMI)}

In last year's edition of this journal, Fan et al. [21] evaluated the feasibility of low frame rate fluoroscopy during primary percutaneous coronary intervention (PPCI) for patients with acute ST elevation myocardial infarction (STEMI) by including 165 consecutive patients. On this study the authors found that low frame rate fluoroscopy at 7.5 frames/s is a safe and feasible strategy for reducing radiation during PPCI. 
On a similar topic, Okuya et al. [22] analyzed the impact of tissue protrusion after coronary stenting in patients with ST-segment elevation myocardial infarction. They included 104 STEMI patients and analyzed IVUS, coronary angiography and single-photon emission computed tomography myocardial perfusion imaging (SPECT MPI) with 99mTc tetrofosmin. During a median follow-up of 14 months after primary PCI, they demonstrated a significantly higher incidence of major adverse cardiac events in patients with tissue protrusion compared to those without, and concluded that tissue protrusion on IVUS after coronary stenting was associated with poor outcomes in patients with STEMI.

\section{Evaluation of drug-coated balloons (DCB)}

Paramount for the role of DBCs in the coronary scenario, Liu et al. [23] investigated the feasibility, initial safety and efficacy of the SeQuent ${ }^{\circledR}$ Please DCB (B. Braun Melsungen, Germany) for patients with de novo coronary lesions. They included a total of 120 patients (135 lesions), with vessels $\geq 3.0 \mathrm{~mm}$ that were treated with DCB in this singlecenter prospective observational study, and demonstrated the feasibility, initial safety, and efficacy of coronary intervention using this DCB. The study also highlighted that the coronary dissection (Type A-C) post DCB treatment occurs frequently but is safe at a 12 months follow up.

\section{Evaluation of bioresorbable vascular scaffolds (BVS)}

The BVS are still a hot topic in the research field and there were many publications in the journal this last year. Tenekecioglu et al. [24] assessed the post-implantation shear stress as an emerging tool for differentiation of BVS. They investigated the in-vivo effect of strut thickness and strut protrusion on endothelial wall shear stress (ESS) distribution in ArterioSorb ${ }^{\circledR}$ Absorbable Drug-Eluting Scaffold (ArterioSorb) and Absorb everolimus-eluting Bioresorbable Vascular Scaffold (Absorb®, Abbott Vascular); devices with similar morphology (quadratic structure) but different thickness, including 4591 struts in the analyzed 477 cross-sections in Absorb ${ }^{\circledR}$ (strut thickness $=157 \mu \mathrm{m}$ ) and 3105 struts in 429 cross-sections in ArterioSorb $\AA$ (strut thickness $=95 \mu \mathrm{m}$ ) for the protrusion analysis. They concluded that ArterioSorb ${ }^{\circledR}$ is associated with a more favorable ESS distribution compared to the Absorb ${ }^{\circledR}$, and attributed this differences to different strut thickness/strut protrusion that has significant effect on shear stress distribution.

On a similar topic, Tijssen et al. [25] investigated the clinical outcomes based on the sizing of the device related to the maximal lumen diameter (Dmax) measured by quantitative coronary angiography (QCA) in Absorb BVS ${ }^{\circledR}$ and Xience ${ }^{\circledR}$ EES treated lesions in the AIDA trial. They concluded that there were no differences in event rates between oversized and non-oversized groups in lesions treated with Xience EES. There was no significant difference in lesion-oriented outcomes (LOCE) between oversized and non-oversized treated Absorb ${ }^{\circledR}$ BVS and Xience ${ }^{\circledR}$ EES treated lesions. Non-oversized Absorb ${ }^{\circledR}$ BVS implantation was associated with a higher risk of scaffold thrombosis at complete 2 years follow-up. The majority of very late scaffold thrombosis occurred in non-oversized devices.

Still in this topic, Holck et al. [26] studied procedural findings and early healing response after implantation of a self-apposing BVS in coronary bifurcation lesions. They evaluated feasibility, early healing and self-correcting properties of the Desolve ${ }^{\circledR} 150$ BVS implanted in bifurcation lesions, using the simple, provisional side branch (SB) stenting technique. BIFSORB pilot was a proof-of-concept study enrolling 10 patients with stable angina pectoris and a bifurcation lesion with $\mathrm{SB} \geq 2.5 \mathrm{~mm}$ and less than $50 \%$ diameter stenosis. Procedure and 1-month outcome were evaluated by OCT to assess scaffold performance and healing patterns. They concluded that treatment of bifurcation lesions using Desolve ${ }^{\circledR} 150$ BRS was feasible except for a delivery failure and unsettling thrombus formation behind jailing SB struts, which was completely resolved at 1-month. Self-correcting and even self-expanding properties were confirmed.

Last but not least, Tensol Rodrigues Pereira et al. [27] evaluated the Absorb ${ }^{\circledR}$ BVS performance for overlap versus non-overlap segments in patients with coronary chronic total occlusion. This was a sub analysis from the GHOST-CTO registry. They compared healing and performance between overlap (OL) and non-overlap regions (NOL) of CTO lesions treated with BVS, using OCT. Fourteen patients with overlapping BVS were included from the GHOST-CTO registry, resulting in $25 \mathrm{OL}$ and $38 \mathrm{NOL}$ regions in a 12-month follow-up. They concluded that the OL and NOL segments showed comparable healing in terms of coverage and malapposition. However, neointimal hyperplasia (NIH) was more prominent in the OL region, and commented that the longterm clinical implications of these findings needs further evaluation.

\section{Evaluation of plaque and tissue characteristics}

Highlighting the importance of the interaction between physiology and anatomy, Wu et al. [28] performed a simultaneous evaluation of plaque stability and ischemic potential of coronary lesions in a fluid-structure interaction analysis. They implemented a new computational model of one-way fluid-structure interaction (FSI) to perform a total of 54 analyses in virtual coronary lesion models, based on plaque compositions, arterial remodeling patterns and stenosis morphologies under physiological conditions. They demonstrated that due to a greater lumen dilation and more induced strain, fractional flow reserve 
(FFR) in the lipid-rich lesions was higher than that in fibrous and calcified lesions, concluding that the analysis of FSI to simultaneously evaluate inducible myocardial ischemia and plaque stability may be useful to identify high risk coronary lesions and, ultimately, optimize treatment. Further research is warranted to assess whether a more aggressive treatment may improve the prognosis of patients with non-ischemic, intermediate, and unstable lesions.

On the same topic of plaque and tissue characteristics, Üveges et al. [29] investigated the correlations between three-dimensional angiography parameters of target coronary artery segments and restenosis after stent implantation in 64 patients after single, cobalt chromium platform stent (27 BM stents and 37 DES) implantations in a retrospective analysis $12 \pm 6$ months after the index procedure. Using multivariate logistic regression modelling, they found that the pre-stent arc-to-cord-ratio ( $\mathrm{ACr}$ ) was an independent predictor of in-stent restenosis. Changes of angles at the stent edges following stent implantation correlated with the initial local bending angles. The $\mathrm{ACr}$ predisposed to chronic shear stress in the vessel wall, which may have contributed to the pathological intimal proliferation.

In the peripheral scenario, Tsukiyama et al. [30] evaluated the vascular response to paclitaxel-eluting nitinol selfexpanding stent (Zilver ${ }^{\circledR}$ PTX stent) in superficial femoral artery lesions through the analysis of angioscopic, from the SHIMEJI trial (Suppression of vascular wall Healing after IMplantation of drug Eluting peripheral stent in Japanese patients with the Infra inguinal lesion: serial angioscopic observation). Patients from five centers were enrolled and angioscopic examinations were performed at 2, 6, and 12 months after implantation and evaluated for neointimal coverage (NIC) grade, intra-stent thrombus (IS-Th) grade, and presence of yellow plaque. They concluded that IS-Th and yellow plaque were persistently observed in $62.5 \%$ and $83.3 \%$ cases respectively, at 12 months. An ongoing healing response was observed at 12 months after implantation; however thrombogenic findings were noted, and the authors commented that prolonged dual antiplatelet therapy could potentially enhance the clinical utility of Zilver® PTX.

In an effort to understand unusual coronary tissue healing patterns, Vergara-Martel et al. [31] described an interesting case of very early in-stent neoatherosclerosis assessed by OCT that occurred 54 days after the DES implant. To better characterize the lesion, OCT was performed and showed red thrombus flanked by regions of lipid plaque distinguished by strong OCT signal attenuation, and healthy neointimal stent coverage was also visualized. Due to the impeded visualization of stent struts undoubtedly present in the region, they concluded that the lipid plaque had developed over the stent and was therefore neoatherosclerosis, which is related to late stent failure.
On a bench study, Shimokado et al. [32] performed imaging assessment in coronary artery autopsy comparing frequency-domain optical coherence tomography (FD-OCT) with IVUS, histology and time-domain OCT (TD-OCT), aiming to demonstrate better accuracy of FD-OCT. Images were acquired from 203 segments from 31 coronary arteries obtained from 20 autopsy cadavers. They concluded that FD-OCT achieved high diagnostic accuracy for the classification of coronary plaques comparable to TD-OCT, and suggested that physicians should consider the differences in the ability to classify plaque morphology of OCT of imaging devices when applying their use.

\section{Endothelial shear stress and vessel physiology}

On an effort to understand the impact of vessel morphology on physiology, Zhang et al. [33] assessed a novel approach for automatic flow velocity computation in deriving quantitative flow ratio (QFR) from coronary angiography. They used a provided patient-specific flow for computation of QFR (QFRauto®) that was assessed in all patients enrolled in the FAVOR II China study, and compared with the commercialized QFR computational method based on frame count (QFRcount $\left.{ }^{\circledR}\right)$, using pressure wire-based fractional flow reserve (FFR) as the reference standard in 328 vessels with paired FFR data. They concluded that the automatic computation of patient-specific coronary flow velocity based on coronary angiography is feasible. Assessment of QFR based on this novel approach had good diagnostic accuracy in determining the functional significance of coronary stenosis.

Finally, on the topic of angiography, Hideo-Kajita et al. [34] evaluated the impact of two formulas to calculate percentage diameter stenosis (\%DS) of coronary lesions from stenosis models (phantom lesion model) to actual clinical lesions. They evaluated the difference between QCA assessed in two datasets, phantom lesion models and CAD patients. Ten phantom lesion models (PLMs) and $354 \mathrm{CAD}$ lesions from the FIRST trial were assessed by QCA in different scenarios. They concluded that although the overall means of the formulas provided similar results, significant lesion-level differences were observed. The use of the worst view versus the average of two views provided similar results.

\section{Nuclear cardiology}

In 2019 different excellent paper in the field of nuclear cardiology were published in the journal. In this review we selected a few papers on SPECT and PET imaging for the assessment of coronary artery disease, coronary inflammation, endocarditis and cardio-oncology. 


\section{Cadium-zinc telluride cameras for myocardial SPECT imaging}

Cadmium-zinc telluride (CZT) cameras have introduced significant progress in myocardial perfusion SPECT imaging, offering high-quality images despite lower doses and scan time. Bednarova et al. [35] evaluated the prognostic value of ultra-low dose Thallium CZT SPECT imaging in 366 consecutvie patients. The stress test was performed by bicycle ergometry or regadenoson injection with administration of $0.5 \mathrm{MBq}(0.014 \mathrm{mCi}) \mathrm{Tl}-201$ chloride/ $\mathrm{kg}$ body weight. Stress images were acquired immediately and redistrution images were taken after $3 \mathrm{~h}$. After a mean follow-up of 23 months, $29 \%$ of the 72 patients with ischaemia on baseline SPECT imaging experienced cardiac events as compared to only $7.9 \%$ of patients without ischemia (HR 4.15, 95\%CI 2.30-7.51, $\mathrm{p}<0.0001)$.. The authors conclude that the faster, low-radiation myocardial perfusion SPECT protocol in a CZT camera maintains the ability to risk stratify patients referred for myocardial perfusion imaging, as compared to conventional SPECT imaging. These kind of studies are important the define the net clinical benefit of this new technology in daily practice.

\section{Novel 18F-labeled tracers for PET myocardial perfusion imaging}

Noninvasive evaluation of coronary artery disease is still a challenging task in dialy practice. Advantages of PET imaging include a more reliable quantification of absolute myocardial blood flow, the routine use of computed tomography for attenuation correction, a higher spatiotemporal resultation and a higher count sensitivity. Werner el al. [36] wrote an excellent review on the use of classical PET myocardial perfusion imaging tracers as well as potential novel $18 \mathrm{~F}$-labeled perfusion radiotracers. These later tracers have a longer half-life of $110 \mathrm{~min}$, allowing exercise testing instead of only pharmacological vasodilator stress testing for tracers with much shorter half-life such as rubidium-82, oxygen- 15 water or nitrogen-13 ammonia. Potential other advantages of 18F-labeled tracers are an improved cost-effectiveness due to the use of pre-existing delivery systems and superior imaging qualities.

\section{Imaging of carotid plaques inflammation in statin treated patients}

Kang et al. [37] performed an interesting prospective 3 time points study of 18F-FDG PET/CT imaging of carotid plaques during the early start-up of statin treatment (initiation to 3 months) as well as during the statin treatment (3 months to 1 year) in 19 statin naïve stable angina patients with inflammatory carotid plaques. Patients were treated with atorvastatine $20 \mathrm{mg} /$ day and underwent $18 \mathrm{~F}$ FDG PET/CT scanning at baseline, at 3 months and at 12 months. The primary outcome was the inter-scan $\%$ change in target-to-background ratio ( $\triangle \mathrm{TBR}$ ) within the index carotid vessel. Interstingely, the index vessel $\Delta \mathrm{TBR}$ showed continuous plaque inflammation reduction over 1 year, by $4.4 \%$ $(p=0.015)$ from the initiation to third month and 6.2\% $(p=0.009)$ from the third month to 1 years, respectively, without a correlation with lipid profile change. Similar founding were noted at other sites such as the ascending aorta. This study indicates that the anti-inflammatory effects of statins continue throughout its use up to 1 year, although stable plasma LDL cholesterol levels are reached at 3 months.

\section{WBC SPECT/CT imaging in infective endocarditis}

The diagnosis of infective endocarditis is often difficult and not always straightforward in daily clinical practice. Szot et al. [38] compared the diagnostic performance of $99 \mathrm{mTc}$ hexamethylpropyleneamineoxime (99mTc-HMPAO) labeled leucocytes SPECT/CT with transthoracic echocardiography (TTE) in 40 consecutive patients with suspected infective endocarditis. All patients underwent clinical examination, TTE and 99mTc-HMPAO SPECT/CT at baseline. Final diagnosis of infective endocarditis was established in 14 (35\%) patients after 6 months follow-up. As compared to TTE, 99mTc-HMPAO SPECT/CT showed a lower number of initital fals-positive results with a significantly higher diagnostic accuracy, specificity and positive predictive value. Although initital transesophageal echo data were not reported, this small study inidciates that $99 \mathrm{mTc}-\mathrm{HMPAO}$ SPECT/CT may help during the initial work-up to differentiate infectious and sterile echocardiographic lesions and may reduce significantly the number of misdiagnosed infective endocarditis patients in the "possible infective endocarditis" category by modified Duke Criteria. However, these findings needs confirmation in larger studies, that should include a more comprehensive work-up including transoesophageal echo and potentially FDG PEC/CT and also patients suspected of prothethic valve endocarditis.

\section{Cardiac nuclear imaging in cardio-oncology}

\section{CMR versus MUGA scans in breast cancer patients receiving trastuzumab}

Few studies have compared multiple-gated acquisition (MUGA) scanning with cardiovascular magnetic resonance (CMR) for serial cardiac monitoring of breast cancer patients. Dhir et al. [39] studied 41 patients with HER2 + breast cancer who underwent MUGA, CMR, NT-proBNP and troponin I measurements at baseline and 
at 6,12 and 18 months after trastuzumab initiation. Both modalities demonstrated a significant LVEF decline at 6 and 12 months from baseline, concomitant with increased LV volumes on CMR. Changes in NT-proBNP correlated with changes with LVEDV at 12 and 18 months, and LVESV at 18 months. Changes in troponin-I did not correlate with changes in $\mathrm{LV}$ function or volume at any time point. Importantly, LVEF comparison between MUGA and CMR demonstrated only a weak agreement with wide LVEF agreement limits on Bland-Altman plots (pooled agreement limits $3.0 \pm 6.2$ ). As pointed out by the authors, these data suggest thatn CMR and MUGA LVEF measurements are not interchangeable, warranting selection and use of one modality only for serial monitoring.

\section{FDG PET/CT for differentiating malignancy of the pulmonary artery versus pulmonary thromboembolism}

$\mathrm{Xi}$ et al. [40] published an interesting report on the accuracy of FDG PET/CT for differentiating pulmonary artery malignancy versus pulmonary thromboembolism (PTE). They pooled their own data on 11 patients with pulmonary artery sarcoma and 9 cases of PTE together with data in the literature on 50 patients with malignant pulmonary artery lesions ( 40 cases of pulmonary artery sarcoma and 10 cases of tumor embolism) as well as 22 patients with PTE. Overall, patients with malignant lesions had significantly higher FDG SUVmax values. Based on the pooled analysis of the literature data and their own cases, the AUC for FDG SUVmax to differentiate pulmonary artery malignancy from PTE was 0.996 (95\% CI 0.989-1.000). At a cutoff value of 3.3, the sensitivity, specificity and accuracy were $98 \%, 97 \%$ and $98 \%$ respectively. Based on these pooled data on a rare clinical entity, it can be concluded that FDG SUVmax is an accurate index for determining pulmonary artery malignancy.

\section{Echocardiography}

Echocardiography, in daily clinical practice the cardiologist's best friend: cheap, readily available and versatile is an imaging modality that is well represented in the scientific manuscripts submitted to this Journal. We will highlight some of these publications.

\section{Eyeballing to evaluate right ventricular function}

Schneider et al. [41] evaluated the visual assessment ("eyeballing") of right ventricular function. The complex anatomy and physiology of the right ventricle is a major limitation of visual echocardiographic gradation of right ventricular systolic function. The authors compared visual assessment ("eyeballing") of right ventricular function with gold standard magnetic resonance imaging (MRI)-derived right ventricular ejection fraction. Medical professionals from a range of clinical settings and with varying degrees of echocardiography experience were recruited via an online ultrasound teaching platform. In an anonymized web-based test, 868 participants graded right ventricular function in 10 patients with varying degrees of right ventricular function via "eyeballing" of an right ventricular-focused four-chamber view. Two skills were evaluated: ability to differentiate between normal and reduced right ventricular function; and ability to determine the correct degree of right ventricular systolic dysfunction. For detection of reduced right ventricular function (MRI-right ventricular ejection fraction $<50 \%$ ), sensitivity was $97.1 \%, 96,8 \%, 96.5 \%$, and $95.8 \%$ and specificity was $55.7 \%, 52.8 \%, 54.6 \%$, and $42.5 \%$ for the expert, advanced, intermediate, and beginner groups, respectively. For determination of the correct degree of right ventricular dysfunction, even experienced examiners assigned a diagnosis that was discordant with MRI in $>40 \%$ of cases. In the present cohort, "eyeballing" was associated with excellent sensitivity but poor specificity in terms of differentiation between normal and abnormal right ventricular function. Even among experts, classification of the degree of dysfunction was imprecise. In accordance with current guidelines, the present data suggest that "eyeballing" should be combined with evaluation of other echocardiographic parameters of right ventricular function.

\section{Effects of marathon running on diastolic function}

Roeh et al. [42] studied the effect of marathon running on left ventricular diastolic function using two dimensional and real-time three dimensional ultrasound measurements in a substudy of the BeMaGIC trial. Diastolic function was evaluated by transthoracic echocardiography in 212 healthy male marathon runners in the week prior to, immediately after, $24 \mathrm{~h}$ after and $72 \mathrm{~h}$ after a marathon race. Real time threedimensional echocardiography included maximal and minimal left atrial volume, total left atrial ejection fraction, total left atrial stroke volume, true ejection fraction and atrial stroke volume. After adjustment for possible confounders (heart rate and systolic blood pressure), 2-dimensional parameters of left ventricular inflow decreased from pre- to immediately post-race and returned to baseline within $24 \mathrm{~h}$. $\mathrm{E} / \mathrm{e}^{\prime}$ mean -ratio remained unchanged directly post-race, but was significantly increased during follow-up of 24 and $72 \mathrm{~h}$. Three-dimensional left atrial minimal volume was increased immediately postrace and in the $24 \mathrm{~h}$ follow-up, left atrial maximal volume was increased immediately post-race and in the follow-up of 24 and $72 \mathrm{~h}$. During follow-up of $72 \mathrm{~h}$, but not immediately postrace, true ejection fraction and atrial stroke volume were significantly increased. Both techniques 
revealed acute and prolonged alterations of diastolic left ventricular function. Considering all parameters, the recovery of diastolic left ventricular after a marathon seems to take longer than previously assumed.

The association between physical activity and cardiac performance is dependent on age.

The Copenhagen City Heart Study aimed to test the hypothesis that regular physical activity is associated with improved cardiac function measured by tissue Doppler imaging in the general population [43]. Within a large prospective community-based population study, cardiac function was assessed in 2221 persons by tissue Doppler. Longitudinal displacement, early diastolic velocity (e'), and myocardial performance index was obtained by tissue Doppler. Linear univariable and multivariable regression analyses were performed in relation to age groups $(<50$ years, 50-65 years, $>65$ years) and self-reported level of physical activity: inactivity, light activity, moderate activity and high-level activity. Participants $<50$ years in the most active group had significantly better cardiac performance when compared to all other activity levels. In age $>65$ years, there was a tendency of impaired cardiac function in higher levels of exercise. Interaction analysis revealed that age significantly modified the association between physical activity and cardiac function. The authors found a positive association between higher level of physical activity and improved cardiac function in younger persons ( $<50$ years). In the general population, however, the association interacted with age and amongst persons above 65 years there was a negative association between higher level of physical activity and cardiac function.

\section{Insights into functional mitral regurgitation using the average pixel intensity method}

Kamoen et al. [44] previously introduced and validated the average pixel intensity method for grading mitral regurgitation in a heterogeneous mitral regurgitation population. They now investigated the feasibility and added value of the average pixel intensity method more specifically in 283 patients with functional mitral regurgitation. Transthoracic echocardiography was performed and mitral regurgitation was assessed using the average pixel method and guidelinerecommended parameters, including color Doppler, vena contracta width and proximal isovelocity surface areabased methods. The average pixel intensity method had an applicability of $98 \%$ in this functional mitral regurgitation cohort, which was significantly higher than vena contracta width $(84 \%)$ and proximal isovelocity surface area-based methods (75\%). Overall, the average pixel intensity method had significant correlations with direct parameters of functional mitral regurgitation severity, ejection fraction, atrial and ventricular dimensions, pulmonary pressures and New
York Heart Association class. Based on ROC curves of established functional mitral regurgitation severity cut-offs, an average pixel intensity value of 125 au was considered the optimal cut-off to determine severe mitral regurgitation. Interestingly, this average pixel intensity severity cut-off is similar to the average pixel intensity severity cut-off for mitral regurgitation in degenerative mitral regurgitation, despite different effective regurgitant orifice area/regurgitant volume cut-offs in current ESC guidelines for functional and degenerative mitral regurgitation. The average pixel intensity method is an easy, fast and feasible parameter for grading functional mitral regurgitation and may complement the multiparametric assessment of functional mitral regurgitation in daily clinical practice.

\section{Can stress echocardiography identify patients who will benefit from percutaneous mitral valve repair?}

Velu et al. [45] investigated whether stress echocardiography may improve the selection of patients who will have clinical benefit from percutaneous mitral valve repair with the MitraClip. In total, 39 patients selected for MitraClip implantation underwent preprocedural low-dose stress (dobutamine or handgrip) echocardiography from which stroke volume, ejection fraction and mitral regurgitation grade were measured. Outcome after MitraClip implantation was determined by New York Heart Association classification and Quality of Life questionnaires. Clinical benefit from MitraClip treatment was defined as survival and NYHA class I-II at 6 months follow-up. All seven patients with mitral regurgitation decreasing during stress remained in NYHA III-IV or died within 6 months, while $62 \%$ (18 out of 29) of the patients with stable or increased mitral regurgitation during stress had clinical benefit. Patients with a decreased mitral regurgitation during preprocedural stress echocardiography remained more symptomatic than patients with a stable or increased mitral regurgitation during stress. Stress echocardiography may support patient selection for percutaneous mitral valve repair.

\section{Left ventricular assessment in patients with systemic light chain amyloidosis: a 3-dimensional speckle tracking transthoracic echocardiographic study}

Cardiac involvement in systemic light chain (AL) amyloidosis carries a poor prognosis mainly through involvement of the left ventricular myocardium. Pradel et al. [46] hypothesized that 3D-transthoracic echocardiography coupled with speckle tracking imaging allows earlier detection of left ventricular systolic dysfunction than 2D-echocardiography in light chain amyloidosis and prospectively studied 71 subjects including 58 patients with confirmed light chain amyloidosis 
(mean age $66 \pm 10$ years, $60 \%$ male) and 21 healthy control (mean age $64 \pm 7$ years, $48 \%$ male) from 2011 to 2014 at the University Hospital of Limoges. The patients were divided into three groups according to Mayo Clinic staging and all subjects underwent 2D and 3D-echocardiography at the same setting. Using 2D, there was no significant difference in left ventricular ejection fraction between the groups. In contrast, 3D-echocardiography demonstrated significantly worse left ventricular systolic function in stage II and III patients using 3D left ventricular ejection fraction, global longitudinal strain and global radial strain. Furthermore, stage III patients had significantly worse global circumferential strain and area tracking. Despite an apparently preserved left ventricular ejection fraction by 2D-echocardiography, light chain amyloidosis patients in stage II and III demonstrate evidence of left ventricular systolic dysfunction by 3D imaging using left ventricular imaging and strain analysis. Worse left ventricular involvement by light chain amyloidosis was associated with more impaired 3D-transthoracic left ventricular systolic parameters.

\section{Should we redefine the normal upper limits of left atrial size ?}

Different cut-offs have been proposed for left atrial size. Furthermore, conflicting results have been reported about the influence of age on left atrial size and data on the impact of age on left atrial myocardial function are scarce. D'Ascenzi et al. [47] conducted a systematic literature search to derive reference values for left atrial size and function in healthy subjects and to evaluate the impact of age. The final population consisted of 62,821 subjects. Left atrial volume index did not differ among different age groups. The normal upper limit of left atrial volume index was $24 \mathrm{~mL} / \mathrm{m}^{2}$. Left atrial reservoir function, measured by strain, did not differ among age groups $(38 \pm 3 \%, 32-43 \%$; $p=0.74)$. The current reference values of left atrial volume index should be used with caution when applied to healthy subjects.

\section{Left atrial, ventricular and atrio-ventricular strain in patients with subclinical heart dysfunction}

Arterial hypertension and diabetes mellitus are the most common causes of heart deterioration because of their high prevalence in the population. Cameli et al. [48] evaluated peak left atrial longitudinal strain, left ventricular longitudinal strain and global atrial-ventricular strain, by speckle-tracking echocardiography, in 162 asymptomatic patients with arterial hypertension or/and diabetes and normal left atrial, left ventricular size and ejection fraction, to analyze their capability to detect early subclinical dysfunction. Longitudinal strain, although with lower values in hypertensives, diabetics and both, did not show significant differences between groups. Peak left atrial longitudinal strain and global atrial-ventricular strain were significantly reduced in hypertension $(31.9 \pm 10.3 \%$ and $49.7 \pm 11.2 \%$, respectively) and diabetes $(26.2 \pm 7.1 \%$ and $42.6 \pm 9.8 \%$ ) compared to controls, and even more if the two coexisted $(20.4 \pm 6.5 \%$ and $37.1 \pm 8.4 \%)$. Peak left atrial longitudinal strain had the highest statistical significance and was able to identify subclinical damage independently from longitudinal strain value. Peak left atrial longitudinal strain was reduced in patients with arterial hypertension and/or diabetes mellitus without alteration of standard echo indexes. The value of peak left atrial longitudinal strain was independent from longitudinal strain and was sufficient to identify heart dysfunction in an earlier stage.

\section{Prognostic value of left atrial strain in patients with moderate asymptomatic mitral regurgitation}

For patients with asymptomatic mitral regurgitation, the criteria identifying the groups at higher-risk and their clinical outcome are still uncertain. Therefore, in these patients, optimal time of surgery remains controversial. Cameli et al. [49] compared left atrial strain to other echocardiographic left ventricular and left atrial parameters for the prediction of cardiovascular outcomes in patients with moderate asymptomatic mitral regurgitation. They enrolled 395 patients with primary degenerative moderate asymptomatic mitral regurgitation. Patients were prospectively followed for $3.5 \pm 1.6$ years for the development of cardiovascular events i.e. atrial fibrillation, stroke/transient ischaemic attack, acute heart failure, cardiovascular death. Of 276 patients (mean age $66 \pm 8$ years) who met eligibility criteria, 108 patients had 141 new events. Patients who developed cardiovascular events presented reduced global peak atrial longitudinal strain, reduced left atrial emptying fraction, larger left atrial volume indexed and lower left ventricular strain at baseline $(\mathrm{p}<0.0001)$. With receiving operating characteristics curve analysis, global peak atrial longitudinal strain $<35 \%$ showed the greatest predictive performance (AUC 0.87). Bland-Altman analysis demonstrated good intra- and interobserver agreement with small bias and Kaplan-Meier analysis showed a graded association between peak atrial longitudinal strain and event-free-survival rates. Speckle tracking imaging could provide a useful index, global peak atrial longitudinal strain, to estimate left atrial function in asymptomatic moderate mitral regurgitation in order to optimize timing of surgery before the development of irreversible myocardial dysfunction.

\section{Magnetic resonance imaging}

There were a number of important contributions in cardiovascular MRI (CMR) in 2019. Many of these were related to cardiovascular functional assessment. 
Gong et al. studied early diastolic strain rate measurements in breast cancer patients treated with trastuzumab and found a significant reduction in peak systolic global longitudinal strain (GLS)and global circumferential strain (GCS) following treatment [50]. Soesanto et al. found a moderate correlation between GLS and late gadolinium enhancement (LGE) in patients with rheumatic mitral stenosis [51]. Peak GLS, GCS and global radial strain were found to be independently positively associated with age and negatively associated with body mass index in healthy adults [52]. Cha et al. found that GLS may aid in the erly prediction of adverse left ventricular (LV) remodeling after reperfusion following STEMI [53]. In other work, no significant difference in strain was found for patients with acute myocarditis and preserved ejection fraction (EF) and controls [54]. Decreased blood-myocardium contrast difference on short axis steady state free procession images was found to affect the results of threshold-based software for functional evaluation [55]. CMR and multiple-gated acquisition (MUGA) scanning were compared in breast cancer patients treated with trastuzumab and found to not provide interchangeable functional results [56]. In a study of patients with ischemic mitral regurgitation, right ventricular (RV) dysfunction was associated with greater mitral regurgitation, lower LV EF, larger global ischemic burden and infarct size [57]. Chew et al. found that mitral regurgitation improves in the majority of their patients following transcatheter aortic valve replacement [58]. Intrathoracic fat volume was found to be associated with contractile dysfunction in patients with coronary artery disease [59]. RV EF and RV strain analysis were found to help differential arrhythmogenic RV cardiomyopathy from athlete's heart [60]. Pre-ablation atrial volume was found to be less in patient having successful therapy [61]. Different phenotypes of bicuspid aortic valve were found to have different stiffness as assessed by pulse wave velocity on CMR [62].

There were a number of new publications relating to mapping and extra cellular volume (ECV). Lui et al. found increased LV myocardial $\mathrm{T} 1$ and reduced strain in patients with hypothyroidism [63]. Dastidar et al. found that native T1 mapping correlated well with LGE in patients with transmural myocardial infarction and could differentiate normal, viable and nonviable segments [64]. In related work van Assen et al. found that T1 reactivity could discriminate between normal, ischemic and infarcted myocardium [65]. Combined T1 mapping and tissue tracking analysis was found to predict the severity of ischemic myocardial injury following acute STEMI [66]. ECV was found to be an independent short-term predictor of major adverse cardiovascular events (MACE) [67]. ECV was also found to aid in risk stratification of patients with clinically suspected myocarditis [68]. In a literature review and meta-analysis Blissett et al. found that parametric mapping useful for the diagnosis of myocarditis [69]. Baseline LV dysfunction and the presence of LGE were predictive of MACE. Differences in $\mathrm{T} 2 *$ values were found in various myocardial segments in patients being evaluated for myocardial iron deposition [70].

Feistritzer et al. found similar outcomes for smokers and non-smokers following non-ST-elevation myocardial infarction [71]. The feasibility of using CMR to assess the area at risk following myocardial infarction in the presence of microvascular obstruction was demonstrated in pigs with histologic confirmation [72].

Left atrial fibrosis was found to correlate with the extent of LV LGE and LV strain in patients with hypertrophic cardiomyopathy [73]. The prognostic value of left atrial function assessed with CMR feature tracking was examined in patients with hypertrophic cardiomyopathy [74].

Adenosine triphosphate and adenosine were found to cause similar vasodilatation in patients undergoing stress CMR [75]. Captur et al. showed a 50\% reduction of scan time for motion corrected free breathing LGE while maintaining high imaging quality [76]. A conservative gadolinium contrast administration protocol was found to decrease exposure, cost and time in Duchenne muscular dystrophy patients without change in medical management [77].

Various MR techniques for evaluation of the pulmonary vasculature were reviewed [78]. Ray et al. assessed pulmonary arterial stiffness by CMR and found that it can predict mild pulmonary arterial hypertension [79].

The feasibility of using coronary flow velocity reserve with phase-contrast CMR to assess for stenosis in patients with heavy coronary calcification was demonstrated [80]. Stams et al. validated hemodynamic measurements using 4D flow CMR against invasive measurements in a swine study [81].

There were several CMR contributions concerning congenital heart disease (CHD). RV systolic dysfunction, hypertrophy, and dilatation together with $\mathrm{QRS}$ prolongation and obesity were found to be predictors of ventricular tachycardia [82]. Hu et al. found that tissue tracking is feasible for assessing early ventricular dysfunction in patients post-Fontan with preserved ejection fraction [83]. Ruh et al. demonstrated the feasibility of CMR tissue phase mapping to assess abnormal LV and RV motion ins tetralogy of Fallot patients [84]. Sugrue et al. proposed using a time resolved MRA technique for assessing the pulmonary veins and left atrium in CHD patients [85]. Ebel et al. compared contrast enhanced MRA to 3D steady state free precession (SSFP) for assessment of the RV outflow tract and found that systolic SSFP provided the best results for sizing percutaneous pulmonary valves [86]. In related work, Leonardi et al. examined the role of end systolic SSFP for planning transcatheter pulmonary valve implantation [87].

Ji et al. found that quantification of intraplaque hemorrhage can be predictive of cerebral ischemic events 
after carotid artery stenting [88]. Lu et al. found in a serial high resolution MRI study that older patients have faster progression of coronary artery atherosclerosis than younger ones and that age was independently associated with carotid plaque progression [89]. Contrast enhanced MR venography was found to be comparable to DANTESPACE for the detection of deep venous thrombosis [90].

\section{Computed tomography}

\section{Computed tomography and coronary artery disease (CAD): finally it's in the center of guidelines}

The year for Computer Tomography had been a remarkable one. One of the dominating events were the new ECR Guidelines for the workup of chronic coronary syndromes which comprises a large group of clinical presentations. These include patients with suspected coronary artery disease and stable angina with or without dyspnoe. But also within this category are patients with new onset of heart failure caused by CAD or symptomatic as well as asymptomatic patients more than 1 year after initial diagnosis or revascularization [91]. Coronary artery CT was defined as the diagnostic imaging modality for initial diagnosis of CAD in the setting of chronic coronary syndromes with an additional functional test in the case of ambiguous or nondiagnostic findings. The choice between Coronary CTA or MRI is open to individual decision based on "clinical likelihood, patient characteristics and preference, availability as well as local expertise" [91]. Interestingly enough $\mathrm{FFR}_{\mathrm{CT}}$ was adopted as a valid additional step in diagnosis and decision-making. Another important event in the world of coronary artery disease was the presentation of the first results of the ISCHEMIA-Trial (International Study of Comparative Health Effectiveneess with Medical and Invasive Approaches) on the AHA-Meeting in the fall of 2019. From the imaging perspective it was remarkable that coronary CTA was the gatekeeper for the definition of existing coronary artery disease. The ISCHEMIA-Trial compared the effect of best medical treatment vs. invasive diagnosis and optimal revascularization in patients with stable angina. Follow up of the two groups showed a modest increase in MACE within the invasive group in the first two years after the revascularization. This was counterbalanced by a slight decrease of roughly $2 \%$ in the incidence of MACE from year 3 to 5 after intervention compared to optimal medical treatment (see www.ischemiatrial.org for the set of ppts with study design and initial results). These two events in 2019 causes the community to focus on cardiac $\mathrm{CT}$ as a future workhorse for the diagnosis and decision-making in chronic coronary syndromes.

\section{Coronary CTA}

The German Cardiac CT Register evaluated their data base with 1352 exams to support the superiority of coronary CTA in the detection of obstructive CAD in comparison to stress-ECG [92]. It is another mosaic in the assessment of the value of coronary CTA, though not really surprising. But it is important in the battle with the health insurance companies for proper reimbursement of coronary CTA. Irrespective of the new importance of coronary CTA there are still some issues left to work on. Seppelt et al. compared high-pitchdual-source coronary CTA with the more conventional prospective step-and-shoot approach. High pitch scans resulted in the reduction of radiation dose by $2 / 3$. However image quality and artefact-load was superior in the prospective trigger variant [93]. A similar observation was published by Chen et al. [94]. They looked at small infants not older than one year that were evaluated by CT for cardiac morphology and coronary arteries in complex congenital heart disease: High pitch scanning had the least radiation dose, but image quality was superior with step and shoot prospective trigger. So, when to use high-pitch-scans? For my institution we use it whenever we have either a follow-up or in patients with low risk profiles for CAD. In patients with atrial fibrillation Pan et al. used a prospective trigger-approach with $100 \mathrm{kVp}$ and low contrast media concentration for $270 \mathrm{mg}$ $\mathrm{I} / \mathrm{mL}$ to yield adequate image quality compared to standard protocol with $120 \mathrm{kVp}$ and a concentration of $370 \mathrm{mg} \mathrm{I} / \mathrm{mL}$ [95]. Meanwhile Molloi et al. in a technical paper showed the superiority of a new evaluation of vascular stenoses in small vessel diameter. Their approach to classify stenoses used an estimation of the perfused vessels cross sectional area based on the distribution of Houndsfield-Units instead of contour-based based vessel-boundaries [96].

\section{CT-FFR is a valid option to classify stenoses: current views}

CT-FFR is exclusively offered by HeartFlow. And they do everything to keep it that way. Yoshikawa published an alternative approach which uses slightly different algorithms to circumvent legal issues and enable a local in-house calculation of the CT-FFR for a specific coronary artery territory [97]. Though it is still a research tool it is surely something to look out for in the expected increasing demand for CTFFR in the future. FFRct by Heartflow itself as we all know does not work with every CT-scan. Nozue et al. analyzed their data as to the rejection-rate by HeartFlow. A heart rate above $65 \mathrm{bpm}$ in a 64-MSCT scanner as well as an Agatston score above 400 were prognostic of rejection of the dataset by HeartFlow [98].

Ko et al. correlated CT-FFR, simple coronary CTA and CT-Perfusion with the results from invasive FFR. CT-FFR 
correlated best with invasive FFR for detection of obstructive coronary artery stenoses [99]. In a similar approach Ghekiere et al. looked more closely on intermediate stenoses which are known to be less easy to judge with CTFFR. CT-FFR correlated well with invasive FFR but not so well with semi-quantitatively analyzed MRI perfusion scans [100]. However, both approaches used the invasive evaluation of coronary artery stenosis as the ground-truth of disease. They did not refer to the induced myocardial ischemia that might depend on more than a single coronary artery stenosis. Patel et al. looked into this with a broader perspective: Patients with stable angina received coronary CTA and regadenoson based static CT perfusion. 76 patients were included and $39 \%$ had hemodynamically significant stenosis in CT-FFR in at least on artery. From these 76 patients only $9 \%$ experienced death (1 patient) or revascularization (6 patients) within 3 years. These 7 patients included two cases with discordant findings in CT-FFR and regadenoson static perfusion CT. Sub-endokardial perfusion defects indicated significant CAD in CTperfusion [101]. Interestingly hypodense streak artefacts were the most important source of error in the evaluation of the single-shot perfusion CT-scan. Patel et al. used an excellent visualization strategy to fuse CT-FFR results with 3D CT-Perfusion maps. Their results support that coronary artery stenoses per se do not resemble the extend of disease of the myocardium in CAD. This is similar to Nissen et al. who looked at the DAN-NICAD-1 substudy population that included patients with obstructive CAD shown by coronary CTA and augmented with clinical information and a functional ischemia test - Myocardial scintigraphy or cardiac MRI [102]. 292 patients were evaluated by invasive coronary angiography and conditional invasive FFR. Only on third, 31 percent, of these patients were revascularized based on the findings in invasive cath. Less than half of the revascularized patients had abnormal ischemia tests. But these patients received more complex revascularization procedures. Multi-vessel CAD in CT and positive ischemia tests were predictive of revascularization - not the pattern of angina. But typical angina correlated best with positive ischemia tests. So myocardial disease does matter in CAD. And myocardial ischemia is not always directly linked to epicardial vascular stenoses. Bechsgaard et al. looked at 143 women with angina and positive ischemia test in static perfusion CT but no evidence of CAD in invasive catheter exams [103]. Semiquantitative analysis of the CT myocardial perfusion was done by calculating the relative enhancement from rest to regadenoson-perfusion. Women with angina but without epicardial CAD had significantly lower relative enhancement of the myocardium compared to healthy controls - hypothesized to resemble microvascular disease.

\section{Beyond the coronaries: CT enters myocardial characterization}

CT-perfusion seems to be the next step in cardiac CT-the extension from epicardial vessel imaging to the CT based characterization of the myocardium. And we all hope that with the improving CT-techniques we can revitalize the often proclaimed but never realized "one-stop-shop" for CAD and ischemia: CT should be able to add myocardial characterization with the help of CT myocardial perfusion and iodine-based late enhancement. However, there are some obstacles. Van Assen pointed out the systematic underestimation of myocardial blood flow in shuttle-mode CT perfusion. The quite extensive ex-vivo experiments with pig-hearts in a Langendorff circulation used by Van Assen quantified the underestimation of MBF to be up to $50 \%$ [104]. We know well from other imaging modalities that imaging does not resemble a ground truth but rather a hint to reality with a hopefully well characterized systematic error. So there is still some work to be done for cardiac CT perfusion as well as another interesting topics: CT late enhancement. Hamdy et al. realized an in-vivo optimization study for the optimal time point in CT based late enhancement [105]. The optimal contrast between healthy and infarcted myocardium was found to be at $5 \mathrm{~min}$ after injection of contrast medium. ECV calculation was stable irrespective of the time points choosen-good news for all imagers with a strong ECV affiliation. Hamdy used the shuttle-mode of a dual-source scanner to obtain the late enhancement scans which I found to be an interesting technical feature.

Myocardial characterization also includes the analysis of functional aspects like motion abnormalities and dyssynchrony. What has been successfully established in CMR now comes to cardiac CT-however it mandates retrospective gating acquisitions with its inborn higher effective radiation dose. Ammon et al. as well as Peled et al. looked at the feasibility of feature tracking in CT datasets. While Peled used a prototype for image analysis that was realized in Mathlab [106], Ammon et al. used a commercial available software tool with the necessity to segment the CT data prior to loading it into the final evaluation software tool [107]. Both approaches have definitely still some way to go until feature tracking is a push-button method to be used in daily routine. Shiina et al. published an interesting pilot study of CT based feature tracking and derived calculation of dyssynchrony. They plan to use this appealing approach in the planning of cardiac resynchronization therapies and for imaging its success in patients with congenital heart disease [108].

\section{Tumor, thrombus and infection}

Looking at characterization of cardiomyopathies and inflammatory as well as primary cardiac tumors cardiac CT has 
been used more conservatively for merely morphology resp. basic functional evaluation. In a multi-modality imaging evaluation Huang et al. found CT to be useful in the workup of hypertrophic cardiomyopathy for the exclusion of CAD and for functional information [109]. Arow et al. used CT as a true $3 \mathrm{D}$ assessment of myocardial crypts in the differential diagnosis of myocardial hypertrophy. They observed a tendency of increased incidence of myocardial crypts in the infero-basal position in patients with hypertrophic cardiomyopathy in comparison with myocardial hypertrophy from other causes like arterial hypertension or aortic stenosis [110]. In a series of reviews for the evaluation of tumors in the heart and proximal pulmonary arteries there is common sense that cardiac CT is useful for the detection and initial characterization of ventricular tumors [111] as well as tumors in the proximal pulmonary artery and its extension into the lungs [112], whereas FDG-PET/CT is the modality of choice for the differentiation of benign and malignant tumors of the heart and nearby great vessels $[111,112]$. Difficulties in the exact extension of disease may arise from tumors with close vicinity to RV-myocardium which tends to show high uptake values in non-clamped FDG-PET/CT exams. Irrespective of this possible pitfall, $\mathrm{Xi}$ et al. used a cutoff value of SUV 3.3 for malignancy in FDG-PET-CT to yield a high accuracy of $98 \%$ with sensitivity and specificity in the $90 \mathrm{~s} \%$ [113]. In a systematic evaluation of imaging modalities for the evaluation of left ventricular assist devices (LVAD) the cardiac CT was found to be useful in the detection of bleeding complications and thrombus formation while the FDG-PET was the optimal modality for detection and extend of inflammation. Echocardiography had its strength in the evaluation of failure of the right ventricle after LVAD implantation [114].

\section{Pulmonary vasculature: hypertension, emboli and devices}

CT and pulmonary vessels has gained a new momentum. Especially in view of the rising interventional therapy of chronic embolic pulmonary hypertension (CTPH) new CT technologies like Dual-Energy scans and 3D perfusion maps help to characterize the changes in the pulmonary vascular bed in a quantitative or at least semiquantitative way [115]. A detailed description of the Dual-Energy technique in CT for the evaluation of acute and chronic pulmonary embolism was provided by Rajiah et al. [116]. It is known that new oncologic drugs like dasatinib can induce pulmonary hypertension in the course of therapy. Toya et al. found that the classic ratio of pulmonary trunk diameter to the diameter of the ascending aorta at baseline is predictive of the development of pulmonary hypertension with dasatinib in patients with chronic myelogenous leukemia [117].
Stenoses of the pulmonary vasculature is an important issue in congenital heart disease. Barrera et al. published two studies on this topic. Cardiac CTA has a surprisingly low negative predictive value of $86 \%$ for the detection of pulmonary venous stenoses [118]. PPV and sensitivity and specificity are within the $90 \mathrm{~s}$ in this special population of 0-3 year old patients with congenital heart disease. One of the reasons for the rather low NPV seems to be the very small dimensions of the anatomical structures as well as missing common cut off values for stenoses of pulmonary veins. In another investigation by Barrera et al. the focus was on the evaluation of central vascular stents within pulmonary arteries or thoracic aorta. Most interestingly all stents could be evaluated for position and restenosis in this retrospective analysis of 18 patients ( 32 stents) except for stentgrafts made out of platinum-iridium. These stents caused beamhardening artefacts that made these stents not assessible in cardiac CT [119].

\section{Imaging the aorta}

The race is open for the reduction of the amount of Iodine based contrast agent and radiation dose in the whole-aortic imaging prior to aortic (valve) interventions. Tomizawa applied a recently proposed double-region-of-interest technique for the calculation of contrast-media dose and injection rate for aortic staging with an $80 \mathrm{kVp}$ protocol [120]. Even with single-source scanners a dose of about $30 \mathrm{ml}$ contrast agent under optimal conditions is possible. The double region of interest basically gives an estimate of the bolus propagation time between ascending and descending aorta. As multiple effects of abdominal aorta geometry influence the further propagation of the CM-bolus, this technique works best with thoracic and abdominal aortic scans. Pelvic arteries and below might need a second bolus-timing scan for optimal contrast.

For wide-detector scanners two scan modes are available to combine ECG-gated cardiac scan for coronary arteries with a whole-body aortic staging CTA. The variable helical pitch scan switches from non-gated helical scan of the upper thoracic aorta to ECG-gated helical scan for the heart to again switch to non-gated helical scan for the abdominal and pelvic arteries. As an alternative scan mode the scan starts with an ECG-gated cardiac volume scan followed by a non-gated helical scan of the whole aorta. Both protocols were compared by Shin et al. for mean radiation dose [121]. The ECG gated volume scan followed by helical non-gated aortic scan proved to have a lower mean radiation dose of about $6 \mathrm{mSv}$ compared to about $10 \mathrm{mSv}$ for the variable helical pitch scan.

In terms of follow-up examinations for aortic aneurysm Gao et al. proposed a prototype software that semiautomatically evaluated two aortic CT scans for progress 
of aneurysma formation [122]. It is a quite elegant way to tackle this problem indeed-however it is really a prototype (MeVisLab and Phyton/C++) that includes some manual steps. But even though it reduces processing time by $40 \%$. This is an important step for the aortic imaging community. But look at products for imaging in oncology that keep track of multiple follow-up exams. We are still waiting for similar solutions for the long-term follow up for aortic pathologies with multiple exams.

A side-branch of cardiovascular imaging is the small but extending field of imaging sarcopenia. Soud et al. published a nice meta-analysis of studies using the degree of sarcopenia imaging for the prediction of major adverse events after TAVI-procedure. CT based cross-sectional area of muscles is used as the measurement substrate for the evaluation of sarcopenia [123]. Though not standardized in its details, this technique will increase in importance in the risk assessment for complex therapies including oncology as well as cardiovascular diseases.

\section{What about coronary calcium?}

Coronary calcium scoring is the oldest and most thoroughly evaluated application of cardiac CT. Is it worthwile to revisit this old theme with new ideas? Patel et al. evaluated the radiation dose needed in modern $64 \mathrm{MSCT}$ for the CACscoring to be approximately $1 \mathrm{mSv}$ with increasing values for BMIs larger than $30 \mathrm{~kg} / \mathrm{m}^{2}$ [124]. The impact of new quantitative photon counting $\mathrm{CT}$ detectors on CAC-scoring seems to be a markedly reduced radiation dose while keeping the image quality [125]. In combination with a negative stress echocardiography a zero Agatston score excludes an obstructive CAD and excludes any type of coronary artery disease with an NPV of $92 \%$ [126]. In cases of heavy calcification of the coronary arteries Higashikawa et al. proposed to use fast MRI PC-flow measurements with and without adenosine for the quantification of the coronary flow reserve [127]. The team established an ambitious scan protocol and achieved an NPV of $89 \%$ and PPV of $88 \%$ for the detection of obstructive CAD in an admittedly small number of nine patients. If this seems not too surprising, you might look at a study of Mo et al. that found a correlation of heavy coronary artery calcifications and the extend of sleep apnea [128]. Interestingly enough the correlation was strong for the incidence and duration of sleep apnea and hypopnea but not for the $\mathrm{SpO} 2$ nadir in this cohort.

\section{References}

1. Karabă Y, Rencuzogullari I, Çağdaş M et al (2019) Association of serum uric acid levels with SYNTAX score II and long term mortality in the patients with stable angina pectoris who undergo percutaneous coronary interventions due to multivessel and/or unprotected left main disease. Int J Cardiovasc Imaging 35:1-7. https://doi.org/10.1007/s10554-018-1446-6

2. Rigatelli G, Zuin M, Vassilev D et al (2019) Culotte versus the novel nano-crush technique for unprotected complex bifurcation left main stenting: difference in procedural time, contrast volume and X-ray exposure and 3-years outcomes. Int J Cardiovasc Imaging 35:207-214. https://doi.org/10.1007/s10554-018-1497-8

3. Zasada W, Slezak M, Pociask E et al (2019) In vivo comparison of key quantitative parameters measured with $3 \mathrm{D}$ peripheral angiography, 2D peripheral quantitative angiography and intravascular ultrasound. Int J Cardiovasc Imaging 35:215-223. https ://doi.org/10.1007/s10554-019-01529-5

4. van Es R, van den Broek HT, van der Naald M et al (2019) Validation of a novel stand-alone software tool for image guided cardiac catheter therapy. Int J Cardiovasc Imaging 35:225-235. https://doi.org/10.1007/s10554-019-01541-9

5. Dengke Z, Lan L, Xiangli S et al (2019) Treatment of left accessory cardiac pathway conduction disorders using radiofrequency catheter ablation under the guidance of the Ensite NavX 3D mapping system: a retrospective study. Int J Cardiovasc Imaging 35:387-392. https://doi.org/10.1007/s10554-018-1449-3

6. Fan L, Luo Y, Chen Z et al (2019) A propensity score matched valuation on feasibility of low frame rate fluoroscopy during primary percutaneous coronary intervention for patients with STEMI. Int J Cardiovasc Imaging 35:393-399. https://doi. org/10.1007/s10554-018-1475-1

7. Liu Y, Zhang Y, Deng L et al (2019) 12-Month clinical results of drug-coated balloons for de novo coronary lesion in vessels exceeding $30 \mathrm{~mm}$. Int J Cardiovasc Imaging 35:579-586. https ://doi.org/10.1007/s10554-018-1505-Z

8. Zhang Y, Zhang S, Westra J et al (2019) Automatic coronary blood flow computation: validation in quantitative flow ratio from coronary angiography. Int J Cardiovasc Imaging 35:587595. https://doi.org/10.1007/s10554-018-1506-y

9. Tijssen RYG, Kerkmeijer LSM, Katagiri Y et al (2019) The relationship of pre-procedural Dmax based sizing to lesion level outcomes in Absorb BVS and Xience EES treated patients in the AIDA trial. Int J Cardiovasc Imaging 35:1189-1198. https://doi. org/10.1007/s10554-019-01576-y

10. Kishima H, Mine T, Fukuhara E et al (2019) Predictors of left atrial appendage stunning after electrical cardioversion in patients with atrial fibrillation. Int $\mathbf{J}$ Cardiovasc Imaging 35:1549-1555. https://doi.org/10.1007/s10554-019-01592-y

11. Baumann F, Obeid S, Gilhofer T et al (2019) Right coronary artery motion analysis: a novel method to measure right ventricular systolic function by selective coronary angiography. Int J Cardiovasc Imaging 35:1557-1561. https://doi.org/10.1007/ s10554-019-01606-9

12. Wu X, von Birgelen C, Zhang S et al (2019) Simultaneous evaluation of plaque stability and ischemic potential of coronary lesions in a fluid-structure interaction analysis. Int J Cardiovasc Imaging 35:1563-1572. https://doi.org/10.1007/s10554-01901611-y

13. Üveges Á, Jenei C, Kiss T et al (2019) Three-dimensional evaluation of the spatial morphology of stented coronary artery segments in relation to restenosis. Int $\mathbf{J}$ Cardiovasc Imaging 35:1755-1763. https://doi.org/10.1007/s10554-019-01628-3

14. Chung J, Lee KE, Her A et al (2019) Comparison of fractional flow reserve and angiographic characteristics after balloon angioplasty in de novo coronary lesions. Int J Cardiovasc Imaging 35:1945-1954. https://doi.org/10.1007/s10554-019-01649-y

15. Rodriguez-Granillo GA, Carrascosa P, Goldsmit A et al (2019) Invasive coronary angiography findings across the CAD-RADS classification spectrum. Int J Cardiovasc Imaging 35:1955-1961. https://doi.org/10.1007/s10554-019-01654-1 
16. Watarai M, Otsuka M, Yazaki K et al (2019) Applicability of quantitative flow ratio for rapid evaluation of intermediate coronary stenosis: comparison with instantaneous wave-free ratio in clinical practice. Int J Cardiovasc Imaging 35:1963-1969. https ://doi.org/10.1007/s10554-019-01656-Z

17. Hideo-Kajita A, Wopperer S, Beyene SS et al (2019) Impact of two formulas to calculate percentage diameter stenosis of coronary lesions: from stenosis models (phantom lesion model) to actual clinical lesions. Int J Cardiovasc Imaging 35:2139-2146. https://doi.org/10.1007/s10554-019-01672-z

18. Tomaniak M, Kołtowski Ł, Pietrasik A, Rdzanek A, Jąkała J, Proniewska K et al (2019) A serial 3- and 9-year optical coherence tomography assessment of vascular healing response to sirolimus- and paclitaxel-eluting stents. Int J Cardiovasc Imaging 35(1):9-21

19. Gao X-F, Wang Z-M, Wang F, Gu Y, Ge Z, Kong X-Q et al (2019) Intravascular ultrasound guidance reduces cardiac death and coronary revascularization in patients undergoing drugeluting stent implantation: results from a meta-analysis of 9 randomized trials and 4724 patients. Int J Cardiovasc Imaging 35(2):239-247

20. Nakamura S, Kimura S, Nakagama S, Misawa T, Mizusawa M, Hayasaka K et al (2019) Impact of lesion angle on optical coherence tomography findings and clinical outcomes after drugeluting stent implantation in curved vessels. Int J Cardiovasc Imaging 35(12):2147-2155

21. Fan L, Luo Y, Chen Z, Cai W, Dong X, Lin C et al (2019) A propensity score matched valuation on feasibility of low frame rate fluoroscopy during primary percutaneous coronary intervention for patients with STEMI. Int J Cardiovasc Imaging 35(3):393-399

22. Okuya Y, Saito Y, Sakai Y, Ishibashi I, Kobayashi Y (2019) Impact of tissue protrusion after coronary stenting in patients with ST-segment elevation myocardial infarction. Int J Cardiovasc Imaging 35(3):401-407

23. Liu Y, Zhang Y-J, Deng L-X, Yin Z-Y, Hu T, Wang Q et al (2019) 12-Month clinical results of drug-coated balloons for de novo coronary lesion in vessels exceeding $3.0 \mathrm{~mm}$. Int J Cardiovasc Imaging. 35(4):579-586

24. Tenekecioglu E, Torii R, Katagiri Y, Chichareon P, Asano T, Miyazaki Y et al (2019) Post-implantation shear stress assessment: an emerging tool for differentiation of bioresorbable scaffolds. Int J Cardiovasc Imaging 35(3):409-418

25. Tijssen RYG, Kerkmeijer LSM, Katagiri Y, Kraak RP, Takahashi K, Kogame N et al (2019) The relationship of pre-procedural Dmax based sizing to lesion level outcomes in Absorb BVS and Xience EES treated patients in the AIDA trial. Int J Cardiovasc Imaging 35(7):1189-1198

26. Holck EN, Fox-Maule C, Barkholt TØ, Jakobsen L, Tu S, Maeng $M$ et al (2019) Procedural findings and early healing response after implantation of a self-apposing bioresorbable scaffold in coronary bifurcation lesions. Int $\mathbf{J}$ Cardiovasc Imaging 35(7):1199-1210

27. Pereira GTR, La Manna A, Ichibori Y, Vergara-Martel A, Ramos Nascimento B, Samdani AJ et al (2019) Optical coherence tomography evaluation of the absorb bioresorbable scaffold performance for overlap versus non-overlap segments in patients with coronary chronic total occlusion: insight from the GHOSTCTO registry. Int J Cardiovasc Imaging 35(10):1767-1776

28. Wu X, von Birgelen C, Zhang S, Ding D, Huang J, Tu S (2019) Simultaneous evaluation of plaque stability and ischemic potential of coronary lesions in a fluid-structure interaction analysis. Int J Cardiovasc Imaging 35(9):1563-1572

29. Üveges Á, Jenei C, Kiss T, Szegedi Z, Tar B, Szabó GT et al (2019) Three-dimensional evaluation of the spatial morphology of stented coronary artery segments in relation to restenosis. Int J Cardiovasc Imaging 35(10):1755-1763

30. Tsukiyama Y, Shinke T, Ishihara T, Otake H, Terashita D, Kozuki A et al (2019) Vascular response to paclitaxel-eluting nitinol self-expanding stent in superficial femoral artery lesions: post-implantation angioscopic findings from the SHIMEJI trial (Suppression of vascular wall Healing after IMplantation of drug Eluting peripheral stent in Japanese patients with the Infra inguinal lesion: serial angioscopic observation). Int J Cardiovasc Imaging 35(10):1777-1784

31. Vergara-Martel A, Pizzato PE, Dallan LAP, Pereira GTR, Richmond HCT, Attizzani GF et al (2019) Very early in-stent neoatherosclerosis assessed by optical coherence tomography. Int $\mathbf{J}$ Cardiovasc Imaging 35(10):1793-1794

32. Shimokado A, Kubo T, Matsuo Y, Ino Y, Shiono Y, Shimamura $\mathrm{K}$ et al (2019) Imaging assessment and accuracy in coronary artery autopsy: comparison of frequency-domain optical coherence tomography with intravascular ultrasound and histology. Int J Cardiovasc Imaging 35(10): 1785-1790

33. Zhang Y, Zhang S, Westra J, Ding D, Zhao Q, Yang J et al (2019) Automatic coronary blood flow computation: validation in quantitative flow ratio from coronary angiography. Int J Cardiovasc Imaging 35(4):587-595

34. Hideo-Kajita A, Wopperer S, Beyene SS, Meirovich YF, Melaku GD, Kuku KO et al (2019) Impact of two formulas to calculate percentage diameter stenosis of coronary lesions: from stenosis models (phantom lesion model) to actual clinical lesions. Int J Cardiovasc Imaging 35(12):2139-2146

35. Bednarova V, Kincl V, Kaminek M et al (2019) The prognostic value of ultra low-dose thallium myocardial perfusion protocol using CZT SPECT. Int J Cardiovasc Imaging 35(6):1163-1167

36. Werner R, Chen X, Rowe S et al (2019) Moving nto the next era of PET myocardial perfusion imaging: introduction of novel 18F-labeled tracers. Int J Cardiovasc Imaging 35:569-577

37. Kam M, Kim C, Choo E et al (2019) Anti-inflammatory effect of statin is continously working throughout use: a prospective three time point $18 \mathrm{~F}-\mathrm{FDG}$ PET/CT imaging study. Int J Cardiovas Imaging 35:1745-1753

38. Szot W, Rubis P, Sobelga A et al (2019) 99mTc-HMPAO labeled leukocyte SPECT/CT and transthoracic echocardiography diagnostic value in infective endocarditis. Int J Cardiovasc Imaging 35:749-758

39. Dhir V, Yan A, Nisenbauw R et al (2019) Assessment of left ventricular function by CMR versus MUGA scans in breast cancer patients receiving trastuzumab: a prospective observational study. Int J Cardiovasc Imaging 35:2085-2093

40. Xi X, Gao W, Gong J et al (2019) Value of 18F-FDG PET/CT in differentiating malignancy of pumonary artery from pulmonary thromboembolism: a cohort study and literature review. Int J Cardiovasc Imaging 35:1163-1167

41. Schneider M, Ran H, Aschauer S et al (2019) Visual assessment of right ventricular function by echocardiography: how good are we? Int J Cardiovasc Imaging 35:2001-2008

42. Roeh A, Schuster T, Jung P et al (2019) Two dimensional and real-time three dimensional ultrasound measurements of left ventricular diastolic function after marathon running: results from a substudy of the BeMaGIC trial. Int J Cardiovasc Imaging 35:1861-1869

43. Joseph G, Mogelvang R, Biering-Sørensen T et al (2019) The association between physical activity and cardiac performance is dependent on age: the Copenhagen City Heart Study. Int J Cardiovasc Imaging 35:1249-1258

44. Kamoen V, El Haddad M, De Backer T et al (2019) Insights into functional mitral regurgitation using the average pixel intensity method. Int J Cardiovasc Imaging 35:761-769 
45. Velu JF, Baan J Jr, de Bruin-Bon HACM et al (2019) Can stress echocardiography identify patients who will benefit from percutaneous mitral valve repair? Int J Cardiovasc Imaging 35:645-651

46. Pradel S, Magne J, Jaccard A et al (2019) Left ventricular assessment in patients with systemic light chain amyloidosis: a 3-dimensional speckle tracking transthoracic echocardiographic study. Int J Cardiovasc Imaging 35:845-854

47. D’Ascenzi F, Piu P, Capone V et al (2019) Reference values of left atrial size and function according to age: should we redefine the normal upper limits? Int J Cardiovasc Imaging 35:41-48

48. Cameli M, Mandoli GE, Lisi E et al (2019) Left atrial, ventricular and atrio-ventricular strain in patients with subclinical heart dysfunction. Int J Cardiovasc Imaging 35:249-258

49. Cameli M, Pastore MC, Righini FM et al (2019) Prognostic value of left atrial strain in patients with moderate asymptomatic mitral regurgitation. Int J Cardiovasc Imaging 35:1597-1604

50. Gong IY, Ong G, Brezden-Masley C et al (2019) Early diastolic strain rate measurements by cardiac MRI in breast cancer patients treated with trastuzumab: a longitudinal study. Int $\mathbf{J}$ Cardiovasc Imaging 35:653-662. https://doi.org/10.1007/s 1055 4-018-1482-2

51. Soesanto AM, Desandri DR, Haykal TM, Kasim M (2019) Association between late gadolinium enhancement and global longitudinal strain in patients with rheumatic mitral stenosis. Int $\mathbf{J}$ Cardiovasc Imaging 35:781-789. https://doi.org/10.1007/s 1055 4-018-1511-1

52. Zhang Z, Ma Q, Cao L et al (2019) Correlation between left ventricular myocardial strain and left ventricular geometry in healthy adults: a cardiovascular magnetic resonance-feature tracking study. Int J Cardiovasc Imaging 35:2057-2065. https:// doi.org/10.1007/s10554-019-01644-3

53. Cha MJ, Lee JH, Jung HN et al (2019) Cardiac magnetic resonance-tissue tracking for the early prediction of adverse left ventricular remodeling after ST-segment elevation myocardial infarction. Int J Cardiovasc Imaging 35:2095-2102. https://doi. org/10.1007/s10554-019-01659-w

54. Gatti M, Palmisano A, Faletti R et al (2019) Two-dimensional and three-dimensional cardiac magnetic resonance feature-tracking myocardial strain analysis in acute myocarditis patients with preserved ejection fraction. Int J Cardiovasc Imaging 35:11011109. https://doi.org/10.1007/s10554-019-01588-8

55. Szúcs A, Kiss AR, Suhai FI et al (2019) The effect of contrast agents on left ventricular parameters calculated by a thresholdbased software module: does it truly matter? Int J Cardiovasc Imaging 35:1683-1689. https://doi.org/10.1007/s10554-01901587-9

56. Dhir V, Yan AT, Nisenbaum R et al (2019) Assessment of left ventricular function by CMR versus MUGA scans in breast cancer patients receiving trastuzumab: a prospective observational study. Int J Cardiovasc Imaging 35:2085-2093. https:// doi.org/10.1007/s10554-019-01648-Z

57. Kim J, Alakbarli J, Yum B et al (2019) Tissue-based markers of right ventricular dysfunction in ischemic mitral regurgitation assessed via stress cardiac magnetic resonance and three-dimensional echocardiography. Int J Cardiovasc Imaging 35:683-693. https://doi.org/10.1007/s10554-018-1500-4

58. Chew PG, Dobson LE, Garg P et al (2019) CMR quantitation of change in mitral regurgitation following transcatheter aortic valve replacement (TAVR): impact on left ventricular reverse remodeling and outcome. Int J Cardiovasc Imaging 35:161-170. https://doi.org/10.1007/s10554-018-1441-y

59. Todd A, Satriano A, Fenwick K et al (2019) Intra-thoracic adiposity is associated with impaired contractile function in patients with coronary artery disease: a cardiovascular magnetic resonance imaging study. Int J Cardiovasc Imaging 35:121-131. https://doi.org/10.1007/s10554-018-1430-1

60. Czimbalmos C, Csécs I, Dohy Z et al (2019) Cardiac magnetic resonance based deformation imaging: role of feature tracking in athletes with suspected arrhythmogenic right ventricular cardiomyopathy. Int J Cardiovasc Imaging 35:529-538. https://doi. org/10.1007/s10554-018-1478-y

61. Curta A, Fichtner S, Wakili R et al (2019) Prospective evaluation of left atrial function and late gadolinium enhancement with $3 \mathrm{~T}$ MRI in patients with atrial fibrillation before and after catheter ablation. Int J Cardiovasc Imaging 35:499-504. https:// doi.org/10.1007/s10554-018-1455-5

62. Boonyasirinant T, Rajiah P, Flamm SD (2019) Abnormal aortic stiffness in patients with bicuspid aortic valve: phenotypic variation determined by magnetic resonance imaging. Int J Cardiovasc Imaging 35:133-141. https://doi.org/10.1007/s1055 4-018-1433-y

63. Liu M, Liu W, Zhang P et al (2019) Left ventricular myocardial T1 mapping and strain analysis evaluate cardiac abnormality in hypothyroidism. Int J Cardiovasc Imaging 35:507-515. https:// doi.org/10.1007/s10554-018-1456-4

64. Dastidar AG, Harries I, Pontecorboli G et al (2019) Native T1 mapping to detect extent of acute and chronic myocardial infarction: comparison with late gadolinium enhancement technique. Int J Cardiovasc Imaging 35:517-527. https://doi.org/10.1007/ s10554-018-1467-1

65. van Assen M, van Dijk R, Kuijpers D et al (2019) T1 reactivity as an imaging biomarker in myocardial tissue characterization discriminating normal, ischemic and infarcted myocardium. Int J Cardiovasc Imaging 35:1319-1325. https://doi.org/10.1007/ s10554-019-01554-4

66. Wamil M, Borlotti A, Liu D et al (2019) Combined T1-mapping and tissue tracking analysis predicts severity of ischemic injury following acute STEMI-an Oxford Acute Myocardial Infarction (OxAMI) study. Int J Cardiovasc Imaging 35:1297-1308. https ://doi.org/10.1007/s10554-019-01542-8

67. Chen R, Wang J, Du Z et al (2019) The comparison of shortterm prognostic value of T1 mapping with feature tracking by cardiovascular magnetic resonance in patients with severe dilated cardiomyopathy. Int J Cardiovasc Imaging 35:171-178. https:// doi.org/10.1007/s10554-018-1444-8

68. Gräni C, Bière L, Eichhorn C et al (2019) Incremental value of extracellular volume assessment by cardiovascular magnetic resonance imaging in risk stratifying patients with suspected myocarditis. Int J Cardiovasc Imaging 35:1067-1078. https:// doi.org/10.1007/s10554-019-01552-6

69. Blissett S, Chocron Y, Kovacina B, Afilalo J (2019) Diagnostic and prognostic value of cardiac magnetic resonance in acute myocarditis: a systematic review and meta-analysis. Int J Cardiovasc Imaging 35:2221-2229. https://doi.org/10.1007/s1055 4-019-01674-x

70. Heiss R, Wiesmueller M, Treutlein C et al (2019) Cardiac T2 star mapping: standardized inline analysis of long and short axis at three identical 1.5 T MRI scanners. Int J Cardiovasc Imaging 35:695-702. https://doi.org/10.1007/s10554-018-1503-1

71. Feistritzer H-J, Eitel I, Jobs A et al (2019) Impact of smoking on cardiac magnetic resonance infarct characteristics and clinical outcome in patients with non-ST-elevation myocardial infarction. Int J Cardiovasc Imaging 35:1079-1087. https://doi.org/10.1007/ s10554-019-01556-2

72. Hansen ESS, Pedersen SF, Pedersen SB et al (2019) Validation of contrast enhanced cine steady-state free precession and T2-weighted CMR for assessment of ischemic myocardial areaat-risk in the presence of reperfusion injury. Int $\mathrm{J}$ Cardiovasc Imaging 35:1039-1045. https://doi.org/10.1007/s10554-01901569-x 
73. Latif SR, Nguyen VQ, Peters DC et al (2019) Left atrial fibrosis correlates with extent of left ventricular myocardial delayed enhancement and left ventricular strain in hypertrophic cardiomyopathy. Int J Cardiovasc Imaging 35:1309-1318. https://doi. org/10.1007/s10554-019-01551-7

74. Hinojar R, Zamorano JL, Fernández-Méndez M et al (2019) Prognostic value of left atrial function by cardiovascular magnetic resonance feature tracking in hypertrophic cardiomyopathy. Int J Cardiovasc Imaging 35:1055-1065. https://doi.org/10.1007/ s10554-019-01534-8

75. García-Baizán A, Millor M, Bartolomé P et al (2019) Adenosine triphosphate (ATP) and adenosine cause similar vasodilator effect in patients undergoing stress perfusion cardiac magnetic resonance imaging. Int J Cardiovasc Imaging 35:675-682. https ://doi.org/10.1007/s10554-018-1494-y

76. Captur G, Lobascio I, Ye Y et al (2019) Motion-corrected freebreathing LGE delivers high quality imaging and reduces scan time by half: an independent validation study. Int J Cardiovasc Imaging 35:1893-1901. https://doi.org/10.1007/s10554-01901620-X

77. Lang SM, Alsaied T, Moore RA et al (2019) Conservative gadolinium administration to patients with Duchenne muscular dystrophy: decreasing exposure, cost, and time, without change in medical management. Int J Cardiovasc Imaging 35:2213-2219. https://doi.org/10.1007/s10554-019-01670-1

78. Aziz M, Krishnam M, Madhuranthakam AJ, Rajiah P (2019) Update on MR imaging of the pulmonary vasculature. Int J Cardiovasc Imaging 35:1483-1497. https://doi.org/10.1007/s1055 4-019-01603-y

79. Ray JC, Burger C, Mergo P et al (2019) Pulmonary arterial stiffness assessed by cardiovascular magnetic resonance imaging is a predictor of mild pulmonary arterial hypertension. Int J Cardiovasc Imaging 35:1881-1892. https://doi.org/10.1007/s1055 4-018-1397-y

80. Higashikawa T, Ichikawa Y, Ishida M et al (2019) Assessment of coronary flow velocity reserve with phase-contrast cine magnetic resonance imaging in patients with heavy coronary calcification. Int J Cardiovasc Imaging 35:897-905. https://doi.org/10.1007/ s10554-019-01531-x

81. Stam K, Chelu RG, van der Velde N et al (2019) Validation of 4D flow CMR against simultaneous invasive hemodynamic measurements: a swine study. Int J Cardiovasc Imaging 35:1111-1118. https://doi.org/10.1007/s10554-019-01593-x

82. Beurskens NEG, Hagdorn QAJ, Gorter TM et al (2019) Risk of cardiac tachyarrhythmia in patients with repaired tetralogy of Fallot: a multicenter cardiac MRI based study. Int J Cardiovasc Imaging 35:143-151. https://doi.org/10.1007/s1055 4-018-1435-9

83. Hu L, Sun A, Guo C et al (2019) Assessment of global and regional strain left ventricular in patients with preserved ejection fraction after Fontan operation using a tissue tracking technique. Int J Cardiovasc Imaging 35:153-160. https://doi.org/10.1007/ s10554-018-1440-z

84. Ruh A, Sarnari R, Berhane H et al (2019) Impact of age and cardiac disease on regional left and right ventricular myocardial motion in healthy controls and patients with repaired tetralogy of fallot. Int J Cardiovasc Imaging 35:1119-1132. https://doi. org/10.1007/s10554-019-01544-6

85. Sugrue G, Cradock A, McGee A et al (2019) Subtraction of timeresolved magnetic resonance angiography images improves visualization of the pulmonary veins and left atrium in adults with congenital heart disease: a novel post-processing technique. Int J Cardiovasc Imaging 35:1339-1346. https://doi.org/10.1007/ s10554-019-01585-x

86. Ebel S, Gottschling S, Buzan MTA et al (2019) 3D-assessment of RVOT dimensions prior percutaneous pulmonary valve implantation: comparison of contrast-enhanced magnetic resonance angiography versus 3D steady-state free precession sequence. Int J Cardiovasc Imaging 35:1453-1463. https://doi. org/10.1007/s10554-019-01578-w

87. Leonardi B, Secinaro A, Perrone MA et al (2019) Role of cardiovascular magnetic resonance end-systolic 3D-SSFP sequence in repaired tetralogy of Fallot patients eligible for transcatheter pulmonary valve implantation. Int J Cardiovasc Imaging 35:1525-1533. https://doi.org/10.1007/s10554-01901630-9

88. Ji A, Lv P, Dai Y et al (2019) Associations between carotid intraplaque hemorrhage and new ipsilateral ischemic lesions after carotid artery stenting: a quantitative study with conventional multi-contrast MRI. Int J Cardiovasc Imaging 35:1047-1054. https://doi.org/10.1007/s10554-018-01521-5

89. Lu M, Peng P, Qiao H et al (2019) Association between age and progression of carotid artery atherosclerosis: a serial high resolution magnetic resonance imaging study. Int J Cardiovasc Imaging 35:1287-1295. https://doi.org/10.1007/s10554-019-01538-4

90. Zhuang G, Tang C, He X et al (2019) DANTE-SPACE: a new technical tool for DVT on 1.5T MRI. Int J Cardiovase Imaging 35:2231-2237. https://doi.org/10.1007/s10554-019-01675-w

91. Knuuti J, Wijns W, Saraste A, Capodanno D, Barbato E, FunckBrentano C, Prescott E, Storey RF, Deaton C, Cuisset T, Agewall S, Dickstein K, Edvardsen T, Escaned J, Gersh BJ, Svitil P, Gilard M, Hasdai D, Hatala R, Mahfoud F, Masip J, Muneretto C, Valgimigli M, Achenbach S, Bax JJ (2020) 2019 ESC Guidelines for the diagnosis and management of chronic coronary syndromes. Eur Heart J 41(3):407-477

92. Barth S, Marwan M, Hausleiter J, Moshage W, Korosoglou G, Leber A, Schmermund A, Gohlke H, Bruder O, Dill T, Schröder S, Kerber S, Hamm K, Gietzen F, Schneider S, Senges J, Achenbach S (2019) Coronary computed tomography angiography (CCTA) in patients with suspected stable coronary artery disease (CAD): diagnostic impact and clinical consequences in the German Cardiac CT Registry depending on stress test results. Int J Cardiovasc Imaging 35(4):741-748

93. Seppelt D, Kolb C, Kühn JP, Speiser U, Radosa CG, Hoberück S, Hoffmann RT, Platzek I (2019) Comparison of sequential and high-pitch-spiral coronary CT-angiography: image quality and radiation exposure. Int J Cardiovasc Imaging 35(7):1379-1386

94. Chen B, Zhao S, Gao Y, Cheng Z, Duan Y, Das P, Wang X (2019) Image quality and radiation dose of two prospective ECGtriggered protocols using 128-slice dual-source CT angiography in infants with congenital heart disease. Int J Cardiovasc Imaging 35(5):937-945

95. Pan Y, Huang Q, Zhu Y, Zou X, Chu H, Du X, Li A, Bu S (2019) Diagnosis of coronary artery disease in patients with atrial fibrillation using low tube voltage coronary $\mathrm{CT}$ angiography with isotonic low-concentration contrast agent. Int J Cardiovasc Imaging 35(12):2239-2248

96. Molloi S, Johnson T, Lipinski J, Ding H, Hubbard L (2019) A phantom based evaluation of vessel lumen area quantification for coronary CT angiography. Int J Cardiovasc Imaging 35(3):551-557

97. Yoshikawa Y, Nakamoto M, Nakamura M, Hoshi T, Yamamoto E, Imai S, Kawase Y, Okubo M, Shiomi H, Kondo T, Matsuo H, Kimura T, Saito N (2020) On-site evaluation of CT-based fractional flow reserve using simple boundary conditions for computational fluid dynamics. Int J Cardiovasc Imaging 36(2):337-346

98. Nozue T, Takamura T, Fukui K, Hibi K, Kishi S, Michishita I (2019) Assessment of factors associated with measurability of fractional flow reserve derived from coronary computed tomography angiography in type 2 diabetic patients with intermediate coronary artery stenosis. Int J Cardiovasc Imaging 35(2):359-365 
99. Ko BS, Linde JJ, Ihdayhid AR, Norgaard BL, Kofoed KF, Sørgaard M, Adams D, Crossett M, Cameron JD, Seneviratne SK (2019) Non-invasive CT-derived fractional flow reserve and static rest and stress CT myocardial perfusion imaging for detection of haemodynamically significant coronary stenosis. Int $\mathbf{J}$ Cardiovasc Imaging 35(11):2103-2112

100. Ghekiere O, Bielen J, Leipsic J, Dewilde W, Mancini I, Hansen D, Dendale P, Nchimi A (2019) Correlation of FFR-derived from $\mathrm{CT}$ and stress perfusion CMR with invasive FFR in intermediate-grade coronary artery stenosis. Int J Cardiovasc Imaging 35(3):559-568

101. Patel AR, Maffessanti F, Patel MB, Kebed K, Narang A, Singh A, Medvedofsky D, Zaidi SJ, Mediratta A, Goyal N, Kachenoura N, Lang RM, Mor-Avi V (2019) Hemodynamic impact of coronary stenosis using computed tomography: comparison between noninvasive fractional flow reserve and 3D fusion of coronary angiography with stress myocardial perfusion. Int J Cardiovase Imaging 35(9):1733-1743

102. Nissen L, Winther S, Westra J, Ejlersen JA, Isaksen C, Rossi A, Holm NR, Urbonaviciene G, Gormsen LC, Madsen LH, Christiansen EH, Maeng M, Knudsen LL, Frost L, Brix L, Bøtker HE, Petersen SE, B $\emptyset$ ttcher M (2019) Influence of Cardiac CT based disease severity and clinical symptoms on the diagnostic performance of myocardial perfusion. Int J Cardiovasc Imaging 35(9):1709-1720

103. Bechsgaard DF, Gustafsson I, Michelsen MM, Mygind ND, Raft KF, Linde JJ, Kofoed KF, Lin FY, Min JK, Prescott E, Hove JD (2020) Evaluation of computed tomography myocardial perfusion in women with angina and no obstructive coronary artery disease. Int J Cardiovasc Imaging 36(2):367-382

104. van Assen M, Pelgrim GJ, Slager E, van Tuijl S, Schoepf UJ, Vliegenthart R, Oudkerk M (2019) Low CT temporal sampling rates result in a substantial underestimation of myocardial blood flow measurements. Int J Cardiovasc Imaging 35(3):539-547

105. Hamdy A, Kitagawa K, Goto Y, Yamada A, Nakamura S, Takafuji M, Nagasawa N, Sakuma H (2019) Comparison of the different imaging time points in delayed phase cardiac CT for myocardial scar assessment and extracellular volume fraction estimation in patients with old myocardial infarction. Int J Cardiovasc Imaging 35(5):917-926

106. Peled Z, Lamash Y, Carasso S, Fischer A, Agmon Y, Mutlak D, Aronson D, Bolotin G, Lessick J (2020) Automated 4-dimensional regional myocardial strain evaluation using cardiac computed tomography. Int J Cardiovasc Imaging 36(1):149-159

107. Ammon F, Bittner D, Hell M, Mansour H, Achenbach S, Arnold M, Marwan M (2019) CT-derived left ventricular global strain: a head-to-head comparison with speckle tracking echocardiography. Int J Cardiovasc Imaging 35(9):1701-1707

108. Shiina Y, Inai K, Takahashi T, Shimomiya Y, Nagao M (2020) Clinical impact of cardiac computed tomography derived threedimensional strain for adult congenital heart disease: a pilot study. Int J Cardiovasc Imaging 36(1):131-140

109. Huang G, Fadl SA, Sukhotski S, Matesan M (2020) Apical variant hypertrophic cardiomyopathy "multimodality imaging evaluation". Int J Cardiovasc Imaging 36(3):553-561

110. Arow Z, Nassar M, Monakier D, Assali A, Vaknin-Assa H, Kornowski R, Hamdan A (2019) Prevalence and morphology of myocardial crypts in normal and hypertrophied myocardium by computed tomography. Int J Cardiovasc Imaging 35(7):1347-1355

111. Lemasle M, Lavie Badie Y, Cariou E, Fournier P, Porterie J, Rousseau H, Petermann A, Hitzel A, Carrié D, Galinier M, Marcheix B, Lairez O (2020) Contribution and performance of multimodal imaging in the diagnosis and management of cardiac masses. Int J Cardiovasc Imaging. https://doi.org/10.1007/s1055 4-020-01774-z
112. Wyler von Ballmoos MC, Chan EY, Reardon MJ (2019) Imaging and surgical treatment of primary pulmonary artery sarcoma. Int J Cardiovasc Imaging 35(8):1429-1433

113. Xi XY, Gao W, Gong JN, Guo XJ, Wu JY, Yang YH, Yang MF (2019) Value of (18)F-FDG PET/CT in differentiating malignancy of pulmonary artery from pulmonary thromboembolism: a cohort study and literature review. Int J Cardiovasc Imaging 35(7):1395-1403

114. Li X, Kondray V, Tavri S, Ruhparwar A, Azeze S, Dey A, Partovi S, Rengier F (2019) Role of imaging in diagnosis and management of left ventricular assist device complications. Int J Cardiovasc Imaging 35(7):1365-1377

115. Rengier F, Melzig C, Derlin T, Marra AM, Vogel-Claussen J (2019) Advanced imaging in pulmonary hypertension: emerging techniques and applications. Int J Cardiovasc Imaging 35(8):1407-1420

116. Rajiah P, Tanabe Y, Partovi S, Moore A (2019) State of the art: utility of multi-energy CT in the evaluation of pulmonary vasculature. Int J Cardiovasc Imaging 35(8):1509-1524

117. Toya T, Nagatomo Y, Kagami K, Yukino M, Yasuda R, Namba T, Ido Y, Kobayashi S, Masaki N, Yada H, Kimura F, Adachi T (2019) Computed tomography-measured pulmonary artery to aorta ratio and EUTOS score for detecting dasatinib-induced pulmonary arterial hypertension. Int J Cardiovasc Imaging 35(8):1435-1442

118. Barrera CA, Saul D, Rapp JB, Smith CL, White AM, Biko DM, Otero HJ (2020) Diagnostic performance of CT angiography to detect pulmonary vein stenosis in children. Int J Cardiovasc Imaging 36(1):141-147

119. Barrera CA, Otero HJ, White AM, Saul D, Biko DM (2019) Image quality and radiation dose of ECG-triggered High-Pitch Dual-Source cardiac computed tomography angiography in children for the evaluation of central vascular stents. Int J Cardiovasc Imaging 35(2):367-374

120. Tomizawa N, Ito S, Nakao T, Arakawa H, Yamamoto K, Inoh S, Nojo T, Nakamura S (2019) Aortic CT angiography using the double region of interest timing bolus technique: feasibility of $80 \mathrm{kVp}$ scanning in lean patients. Int J Cardiovasc Imaging 35(11):2113-2121

121. Shin N, Kim SM, Choe YH (2019) Protocol using wide-detector CT with single contrast injection for the aorta and coronary artery: variable helical pitch versus volume scan following helical scan. Int J Cardiovasc Imaging 35(10):1935-1942

122. Gao X, Boccalini S, Kitslaar PH, Budde RPJ, Tu S, Lelieveldt BPF, Dijkstra J, Reiber JHC (2019) A novel software tool for semi-automatic quantification of thoracic aorta dilatation on baseline and follow-up computed tomography angiography. Int J Cardiovasc Imaging 35(4):711-723

123. Soud M, Alahdab F, Ho G, Kuku KO, Cejudo-Tejeda M, Hideo-Kajita A, de Araujo Gonçalves P, Teles RC, Waksman R, Garcia-Garcia HM (2019) Usefulness of skeletal muscle area detected by computed tomography to predict mortality in patients undergoing transcatheter aortic valve replacement: a meta-analysis study. Int J Cardiovasc Imaging 35(6):1141-1147

124. Patel AA, Fine J, Naghavi M, Budoff MJ (2019) Radiation exposure and coronary artery calcium scans in the society for heart attack prevention and eradication cohort. Int J Cardiovasc Imaging 35(1): 179-183

125. Symons R, Sandfort V, Mallek M, Ulzheimer S, Pourmorteza A (2019) Coronary artery calcium scoring with photon-counting $\mathrm{CT}$ : first in vivo human experience. Int J Cardiovasc Imaging 35(4):733-739

126. AlJaroudi W, Mansour MJ, Chedid M, Hamoui O, Asmar J, Mansour L, Chammas E (2019) Incremental value of stress echocardiography and computed tomography coronary calcium scoring 
for the diagnosis of coronary artery disease. Int $\mathrm{J}$ Cardiovasc Imaging 35(6):1133-1139

127. Higashikawa T, Ichikawa Y, Ishida M, Kitagawa K, Hirano T, Sakuma H (2019) Assessment of coronary flow velocity reserve with phase-contrast cine magnetic resonance imaging in patients with heavy coronary calcification. Int J Cardiovasc Imaging 35(5):897-905

128. Mo L, Gupta V, Modi R, Munnur K, Cameron JD, Seneviratne S, Edwards BA, Landry SA, Joosten SA, Hamilton GS, Wong DTL (2020) Severe obstructive sleep apnea is associated with significant coronary artery plaque burden independent of traditional cardiovascular risk factors. Int J Cardiovasc Imaging 36(2):347-355

Publisher's Note Springer Nature remains neutral with regard to jurisdictional claims in published maps and institutional affiliations. 